\title{
Quantification of Fracture Roughness by Change Probabilities and Hurst Exponents
}

\author{
Tim Gutjahr ${ }^{1}$ (D) - Sina Hale ${ }^{2} \cdot$ Karsten Keller $^{1}$ • \\ Philipp Blum $^{2}$. Steffen Winter ${ }^{3}$
}

Received: 19 March 2021 / Accepted: 5 November 2021 / Published online: 17 December 2021

(C) The Author(s) 2021

\begin{abstract}
The objective of the current study is to utilize an innovative method called "change probabilities" for describing fracture roughness. In order to detect and visualize anisotropy of rock joint surfaces, the roughness of one-dimensional profiles taken in different directions is quantified. The central quantifiers, change probabilities, are based on counting monotonic changes in discretizations of a profile. These probabilities, which usually vary with the scale, can be reinterpreted as scale-dependent Hurst exponents. For a large class of Gaussian stochastic processes, change probabilities are shown to be directly related to the classical Hurst exponent, which generalizes a relationship known for fractional Brownian motion. While related to this classical roughness measure, the proposed method is more generally applicable, therefore increasing the flexibility of modeling and investigating surface profiles. In particular, it allows a quick and efficient visualization and detection of roughness anisotropy and scale dependence of roughness.
\end{abstract}

Keywords Fracture roughness - Anisotropy - Change probability · Fractional Brownian motion $\cdot$ Hurst exponent $\cdot$ Scale dependence

Mathematics Subject Classification $60 \mathrm{G} 18 \cdot 60 \mathrm{G} 15$

Tim Gutjahr

gutjahr@math.uni-luebeck.de

1 Institute of Mathematics, University of Luebeck, Ratzeburger Allee 160, 23562 Luebeck, Germany

2 Institute of Applied Geosciences, Karlsruhe Institute of Technology (KIT), Kaiserstr. 12, 76131 Karlsruhe, Germany

3 Institute of Stochastics, Karlsruhe Institute of Technology (KIT), Englerstr. 2, 76131 Karlsruhe, Germany 


\section{Introduction}

Fracture roughness is an omnipresent and important property of natural rock joints. It varies depending on rock type (e.g., Morgan et al. 2013) or formation mechanism (e.g., Xie et al. 1999; Vogler et al. 2017) and essentially controls the mechanical and hydraulic properties of discontinuities (Magsipoc et al. 2020). Contact areas between two rough fracture surfaces influence fracture strength and deformation under normal or shear loadings (Bandis et al. 1983; Tsang and Witherspoon 1983; Power and Durham 1997; Jiang et al. 2006; Brodsky et al. 2016). The effective hydraulic aperture and related parameters such as permeability and flow, however, are determined by the shape of the void space within the fracture, which is dependent both on the roughness of the individual fracture surfaces and on the degree of mismatch between them (Brown 1987; Roux et al. 1993; Zimmerman and Bodvarsson 1996; Boutt et al. 2006; Zhao et al. 2014). Furthermore, a quantitative description of fracture roughness is a prerequisite for creating synthetic input data sets for numerical fluid flow simulations, which ideally match relevant surface characteristics of natural fractures (e.g., Vogler et al. 2017; Magsipoc et al. 2020). In recent works, researchers often use synthetic fractures with self-affine properties (e.g., Kottwitz et al. 2020; Dong and Ju 2020; Seybold et al. 2020; Yu et al. 2020). For example, Liu et al. (2020) simulate anisotropic flow during shearing by using a double-rough-walled fracture model, while others examine the effects of roughness on fracture permeability or breakthrough curves for conservative solutes (Zambrano et al. 2019; Dou et al. 2019). Fracture roughness is therefore linked to rock mechanics and hydrogeology, and also to various geoscientific fields of application, for example geothermal energy, reservoir engineering, or geological disposal of radioactive waste (Li et al. 2019; Stigsson and Mas Ivars 2019).

Numerous parameters and methods have been proposed to characterize fracture roughness qualitatively and quantitatively (Magsipoc et al. 2020), such as the wellknown joint roughness coefficient, JRC (Barton 1973; Barton and Choubey 1977), or various statistical roughness parameters such as $Z_{2}$ (Myers 1962) and $R_{p}$ (El-Soudani 1978). Today, fractal methods are receiving increasing attention for the quantitative description of fracture roughness and are applied to various fracture types from nanometer scale (e.g., Siman-Tov et al. 2013) to kilometer scale, including studies on earthquake rupture traces (Candela et al. 2012) or coastlines (Renard et al. 2013). The application of fractal methods is based on the general assumption that fracture surfaces can be approximately described as self-affine fractals (e.g., Mandelbrot 1985; Brown 1987; Thompson and Brown 1991; Power and Tullis 1991; Schmittbuhl et al. 1993; Odling 1994; Lee and Bruhn 1996).

Fractal theory allows for detailed analysis of fracture surface morphology and enables upscaling of roughness and related fracture properties from experimental to field scale. In this context, the Hurst exponent (or the related parameter, fractal dimension) represents a measure of roughness and characterizes its scaling behavior (Odling 1994; Issa et al. 2003). The Hurst exponent can also be used to investigate the roughness anisotropy of shear fractures or exposed fault surfaces. Generally, different values are obtained depending on the profile orientation with respect to the original direction of movement of the surface. While Candela et al. (2012) and Corradetti et al. (2017) determined Hurst exponents for natural fault surfaces by using two distinct profile 
directions oriented parallel and perpendicular to the slip, Xie et al. (1999) considered different spatial directions and positions on the fracture surface for calculating fractal dimensions.

In many studies, minimal values of the Hurst exponent were found parallel to the slip direction, and increasing values were observed with progressing angular deviation, which implies a higher roughness along the slip direction (e.g., Renard et al. 2006; Candela et al. 2012; Corradetti et al. 2017). In contrast, Xie et al. (1999) and Sagy et al. (2007) observed that the studied fault surfaces were smoother in the slip-parallel direction. In general, these contrasting observations with regard to the directionality of surface roughness may be explained by the diversity of roughness measures used and their interpretation in spite of model violations. In addition, Sagy et al. (2007) showed that natural small-slip and large-slip fault surfaces are each characterized by different geometric features and can therefore differ greatly in roughness along the slip direction.

Various methods are applied for determining fractal parameters, including Fourier power spectrum (e.g., Sagy et al. 2007; Bistacci et al. 2011; Candela et al. 2012; Renard et al. 2013; Corradetti et al. 2017, 2020), variogram analysis (e.g., Huang et al. 1992; McClean and Evans 2002), structure function (e.g., Poon et al. 1992; Odling 1994), or the box counting method (e.g., Malinverno 1990). Regardless of the methodology used, it should be noted that a correct interpretation of fractal parameters requires self-affinity, or at least some kind of scale invariance.

In this study, an innovative roughness measure is proposed which is linked to the order relation of value triples measured along directional fracture profiles. The method is based on the identification of so-called change patterns and subsequent determination of their relative frequency. This change probability serves as a simple and generally scale-dependent roughness measure. Under the assumption of some kind of self-affinity, the method offers a simple way to determine the Hurst exponent. In the classical setting of fractional Brownian motion, which is widely used in modeling rock profiles (e.g., Brown 1987; Huang et al. 1992; Odling 1994; Hsiung et al. 1995), the change probability is directly related to the Hurst exponent, hence also to the autocorrelation function (Coeurjolly 2000). Even beyond this setting, the method offers the possibility of visualizing, detecting, and specifying the morphological anisotropy of shear fractures or fault planes.

The term "change probability" was first used in Sinn and Keller (2011a) in the given context. The idea, however, is far from being new. When considering increments between values instead of the values themselves, changes turn into so-called zerocrossings. Given a sequence of data points, a zero-crossing occurs if two consecutive data points differ in their sign. Indicators and probabilities of zero-crossings have been applied in various fields, such as signal analysis (e.g., Chang et al. 1951; Ewing and Taylor 1969). Further background on zero-crossings can be found in Sinn and Keller (2011a).

It is important to note that change probabilities are invariant under monotonic transformations of a stochastic process. This invariance is interesting from a theoretical viewpoint, because it widens the class of mathematical models to which the proposed methods apply. On the other hand, this invariance is useful from a practical point of view. For example, differently calibrated measuring devices may map the same 
height information of a fracture surface to different values in the resulting data set (e.g., gray-level image or surface profile). One device might also be more sensitive to small differences than another one, so that the actual heights are mapped onto a larger range of values in the resulting image. This makes it rather difficult to compare data. However, since different mappings typically do not change the relative order of the values, change probabilities are not affected by such effects.

The approach presented herein is simple and economical from a computational point of view, as it is essentially based on comparing values at different locations. No heavy computations, as in the estimation of the autocorrelation function or the Fourier spectrum, are therefore needed. Furthermore, the approach is in line with a relatively new general trend in time series analysis and signal processing aiming to avoid using the exact metric values and to concentrate instead on the ordinal structure of the data (e.g., Amigó 2010; Zanin et al. 2012; Amigó et al. 2014).

The present study is structured as follows: Section 2 introduces the concept of change probabilities as the theoretical base for quantifying directional fracture roughness. The relation between change probabilities and the Hurst exponent is also discussed, firstly in the case of fractional Brownian motion, and secondly for a more general class of Gaussian processes. In Sect. 3, a practical implementation of the proposed methods is described. In Sect. 4, the developed algorithm for roughness analysis is applied to natural fracture surfaces. On this basis, the novel method and its performance is compared to other methods. In Sect. 5, the results are discussed. Finally, concluding remarks are provided in Sect. 6.

\section{Methods}

To provide a first insight into the concept of change probability, it is assumed for simplicity that a profile curve - obtained as a linear section of some surface-is described by a real function on some interval $[0, T]$. For some positive $\tau$ being small relative to $T$ and some $t$ such that $0 \leq t$ and $t+2 \tau \leq T$, consider the consecutive values $x(t)$, $x(t+\tau)$, and $x(t+2 \tau)$. There are six possible ways to order these three values, as depicted in Fig. 1. The idea behind the following method is that values on a smooth curve are more likely to be ordered in such a way that the value in the middle is the second largest out of the three, while for values on a rough curve it is more likely that the value in the middle is the smallest or largest. Out of the six possible orders of the three values, there are four for which the middle value is the largest or smallest one (Fig. 1). These four order patterns will be called "change patterns" because in each of them there is a change from an increasing to a decreasing direction or vice versa.

Although the overall aim is the analysis of rock joint surfaces, this section is devoted entirely to the analysis of one-dimensional profiles. Later in Sect. 3, the introduced method is used to study the anisotropy of surfaces. Throughout, one-dimensional profiles of rock surfaces are modeled as realizations of a stochastic process $\left(X_{t}\right)_{t \in[0, \infty[}$, with values in $\mathbb{R}$ defined on some probability space with measure $\mathbb{P}$, where the value $X_{t}$ represents the height of the profile above some reference line. In other words, it is assumed that such profiles can be represented as graphs of functions, which is debatable but seems to be common practice. It is to some extent justified by the fact 


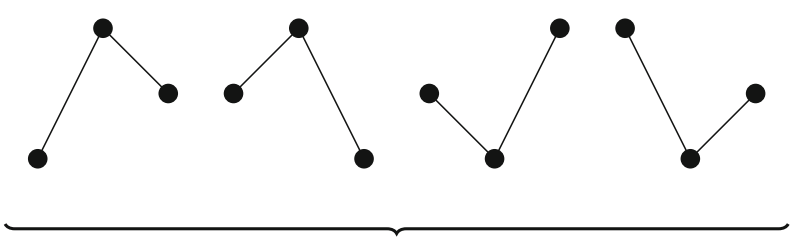

change patterns

Fig. 1 All possible orderings of values at three consecutive points on a profile

that rock surfaces are usually measured using methods such as laser profilometry or white light interferometry, which produce such functional data and ignore the true three-dimensional structure of the surface. It is convenient to assume that the location variable $t \in[0, \infty$ [ has a continuous range. In practice, measurements will only be available at a finite number of locations $t$, and then it is not a serious restriction to set the range to $t \in\{0,1,2, \ldots, n\}$ for some $n \in \mathbb{N}$, as one has the freedom to choose the scale. Details on how to obtain values $x_{0}, x_{1}, \ldots, x_{n}$ for those $t$ from the rock surface are provided in Sect. 3.

\subsection{Roughness by Change Probabilities}

For any stochastic process, a concept of roughness is provided by so-called change probabilities.

Definition 1 Given a stochastic process $\left(X_{t}\right)_{t \in[0, \infty[}$ on some probability space with measure $\mathbb{P}$ and some $\tau \in] 0, \infty[$, the change probability of delay $\tau$ is defined by

$$
p(\tau):=\mathbb{P}\left(X_{0}<X_{\tau}, X_{\tau} \geq X_{2 \tau}\right)+\mathbb{P}\left(X_{0} \geq X_{\tau}, X_{\tau}<X_{2 \tau}\right) .
$$

$p(\tau)$ describes how likely it is to observe one of the four change patterns from Fig. 1 at location 0 . Although $p(\tau)$ is defined for any process, in order to be a useful quantity for the statistics of the process (in particular to become estimable from change counts), it is reasonable to require the process to have some minimal stationarity, namely to require that

$$
\begin{array}{r}
\mathbb{P}\left(X_{t}<X_{t+\tau}, X_{t+\tau} \geq X_{t+2 \tau}\right)+\mathbb{P}\left(X_{t} \geq X_{t+\tau}, X_{t+\tau}<X_{t+2 \tau}\right) \\
\text { does not depend on } t
\end{array}
$$

at least for the locations $t$ and delays $\tau$ of interest. This stationarity of pattern occurrences is a rather weak form of stationarity. It is for instance implied by the common assumption of stationary increments and, in the case of a Gaussian process, in particular by the conditions (A2) and (A3) imposed later on in this section. For this and the following, see for example Bandt and Shiha (2007). 
If $\left(X_{t}\right)_{t \in[0, \infty[}$ is a Gaussian process with zero mean, then $p(\tau)$ is known to be given by the formula

$$
p(\tau)=1-\frac{2}{\pi} \arcsin \left(\sqrt{\frac{\operatorname{Cov}\left(X_{\tau}-X_{0}, X_{2 \tau}-X_{\tau}\right)}{2 \sqrt{\operatorname{Var}\left(X_{\tau}-X_{0}\right) \operatorname{Var}\left(X_{2 \tau}-X_{\tau}\right)}}+\frac{1}{2}}\right),
$$

for all $\tau \in] 0, \infty[$.

Change probabilities are proposed here as a measure of roughness. Below it is argued that they are more generally defined and easier to compute than the Hurst exponent that is typically used. In certain situations there is a direct relation between these two roughness measures. Via their dependence on the delay parameter $\tau$, addressing different scales, change probabilities are able to capture more roughness information than the Hurst exponent.

\subsection{Fractional Brownian Motion, Change Probabilities, and the Hurst Exponent}

As already mentioned, fractional Brownian motion ( $\mathrm{fBm}$ ) is widely used as a model for rock profiles, and the main model parameter, the Hurst exponent $H \in] 0,1$, serves as a quantifier of roughness (see, e.g., Hsiung et al. 1995). Recall that fBm is a Gaussian stochastic process $\left(X_{t}\right)_{t \in[0, \infty[}$ satisfying

$$
\begin{array}{rl}
X_{0}=0 & \mathbb{P} \text {-almost surely, } \\
\mathbb{E}\left(X_{t}\right)=0 & \text { for all } t \in[0, \infty[,
\end{array}
$$

and

$$
\operatorname{Cov}\left(X_{s}, X_{t}\right)=\frac{1}{2}\left(s^{2 H}+t^{2 H}-|t-s|^{2 H}\right) \quad \text { for all } s, t \in[0, \infty[
$$

It has two outstanding properties, the first one being its self-affinity, meaning that, for any $\alpha>0$, the processes

$$
\left(X_{\alpha t}\right)_{t \in[0, \infty[} \text { and }\left(\alpha^{H} X_{t}\right)_{t \in[0, \infty[} \text { have the same distribution. }
$$

This scaling invariance has the consequence that $p(\tau)$ does not depend on $\tau>0$. The second one is that, for any $\tau \in[0, \infty[$,

$$
\operatorname{Cov}\left(X_{t+\tau+1}-X_{t+\tau}, X_{t+1}-X_{t}\right) \quad \text { does not depend on } t \in[0, \infty[
$$

Note that the properties (A2) and (A3) together mean that the increment process $\left(X_{t+1}-X_{t}\right)_{t \in[0, \infty[}$ of the stochastic process $\left(X_{t}\right)_{t \in[0, \infty[}$ is weakly stationary. In the Gaussian case this implies its stationarity. For $\mathrm{fBm}$, the increment process is also known as fractional Brownian noise. 
In order to describe the announced relationship between change probabilities and Hurst exponents, consider the function $h:] 0,1[\rightarrow]-\infty, 1[$ given by

$$
h(x)=1+\log _{2}(\sin (\pi(1-x) / 2))
$$

for $x \in] 0,1[$. The statement characterizing the Hurst exponent in terms of the change probabilities is as follows: If $\left(X_{t}\right)_{t \in[0, \infty[}$ is a fBm with Hurst exponent $\left.H \in\right] 0,1[$, then $h(p(\tau))=H$ for all $\tau \in] 0, \infty[$. This statement goes essentially back to Coeurjolly (2000), who formulated it in terms of zero-crossings in the increment process of a $\mathrm{fBm}$.

It is easy to show that for any $\mathrm{fBm}, p(\tau) \leq 2 / 3$. Moreover, $h\left(\frac{2}{3}\right)=0$ and $h$ maps ]0, $\frac{2}{3}$ [ onto ]0, 1[ in a strictly decreasing way. In particular, the higher the change probability, the lower the Hurst exponent (and thus the rougher the profile). So for the class of fBms, the change probability $p(\tau)$, which does not depend on the scale $\tau$, and the Hurst exponent are equivalent quantifications of roughness. In order to treat the more general situation of scale-dependent roughness, the following definition is introduced.

Definition 2 Given a stochastic process $\left(X_{t}\right)_{t \in[0, \infty[}$ on some probability space with measure $\mathbb{P}$ and some $\tau \in] 0, \infty$ [, the Hurst exponent of delay $\tau$ is defined by $H(\tau)=$ $h(p(\tau))$, where $h$ is as in (3).

Again it is reasonable to assume at least stationarity of pattern occurrences here as introduced in (1). Basically, the Hurst exponent of delay $\tau$ is nothing more than a reinterpretation of the change probability of delay $\tau$. However, it allows a unified discussion of roughness for a large class of stochastic processes in the established framework and an easier comparison with the commonly considered Hurst exponent $H$ (see (5)), whenever it is defined.

\subsection{Generalizing Results to a Wider Class of Gaussian Processes}

The self-affinity of $\mathrm{fBm}$ results in the same change probability $p(\tau)$ (and thus in the same Hurst exponent $H(\tau)$ ) at all delays $\tau$. This kind of scale invariance of roughness is a relatively strong property. It is generally not completely compatible with realworld surface profile data, but interesting from a modeling viewpoint. In order to gain more flexibility on the modeling side, Gaussian processes with asymptotically stabilizing roughness will be considered and investigated, for which the roughness (in the sense of change probabilities) is allowed to vary with the scale. The relation to the Hurst exponent, which ultimately is an asymptotic concept capturing the long-range behavior of a process, will be preserved in the form of an asymptotic equality. This relation is based on the following fundamental characterization of the Hurst exponent of delay $\tau$ (and thus of the change probability $p(\tau)$ ) in terms of variance scaling. 
Proposition 1 Let $\left(X_{t}\right)_{t \in[0, \infty[}$ be a Gaussian stochastic process satisfying (A1) and (A2). If $0<\operatorname{Var}\left(X_{\tau}\right)=\operatorname{Var}\left(X_{2 \tau}-X_{\tau}\right)$ for some $\tau>0$, then

$$
H(\tau)=\frac{1}{2} \log _{2}\left(\frac{\operatorname{Var}\left(X_{2 \tau}\right)}{\operatorname{Var}\left(X_{\tau}\right)}\right) .
$$

Proof Recall formula (2) for the change probability of a Gaussian process. Applying the transformation $h$ to $p(\tau)$ yields

$$
\begin{aligned}
H(\tau)=h(p(\tau)) & =1+\frac{1}{2} \log _{2}\left(\frac{\operatorname{Cov}\left(X_{\tau}-X_{0}, X_{2 \tau}-X_{\tau}\right)}{2 \sqrt{\operatorname{Var}\left(X_{\tau}-X_{0}\right) \operatorname{Var}\left(X_{2 \tau}-X_{\tau}\right)}}+\frac{1}{2}\right) \\
& =\frac{1}{2}+\frac{1}{2} \log _{2}\left(\frac{\operatorname{Cov}\left(X_{\tau}-X_{0}, X_{2 \tau}-X_{\tau}\right)}{\sqrt{\operatorname{Var}\left(X_{\tau}-X_{0}\right) \operatorname{Var}\left(X_{2 \tau}-X_{\tau}\right)}}+1\right) \\
& =\frac{1}{2}+\frac{1}{2} \log _{2}\left(\frac{\operatorname{Cov}\left(X_{\tau}, X_{2 \tau}-X_{\tau}\right)}{\operatorname{Var}\left(X_{\tau}\right)}+1\right) \\
& =\frac{1}{2}+\frac{1}{2} \log _{2}\left(\frac{\operatorname{Cov}\left(X_{\tau}, X_{2 \tau}\right)-\operatorname{Cov}\left(X_{\tau}, X_{\tau}\right)}{\operatorname{Var}\left(X_{\tau}\right)}+1\right) \\
& =\frac{1}{2}+\frac{1}{2} \log _{2}\left(\frac{\operatorname{Cov}\left(X_{\tau}, X_{2 \tau}\right)}{\operatorname{Var}\left(X_{\tau}\right)}\right) .
\end{aligned}
$$

By the assumptions, one has

$$
\operatorname{Var}\left(X_{\tau}\right)=\operatorname{Var}\left(X_{2 \tau}-X_{\tau}\right)=\operatorname{Var}\left(X_{2 \tau}\right)-2 \operatorname{Cov}\left(X_{2 \tau}, X_{\tau}\right)+\operatorname{Var}\left(X_{\tau}\right)
$$

which is equivalent to

$$
\operatorname{Cov}\left(X_{\tau}, X_{2 \tau}\right)=\frac{1}{2} \operatorname{Var}\left(X_{2 \tau}\right)
$$

Hence

$$
H(\tau)=\frac{1}{2}+\frac{1}{2} \log _{2}\left(\frac{\operatorname{Var}\left(X_{2 \tau}\right)}{2 \operatorname{Var}\left(X_{\tau}\right)}\right)=\frac{1}{2} \log _{2}\left(\frac{\operatorname{Var}\left(X_{2 \tau}\right)}{\operatorname{Var}\left(X_{\tau}\right)}\right)
$$

Note that if the process $\left(X_{t}\right)$ satisfies condition (A3), then the assumption $\operatorname{Var}\left(X_{\tau}\right)=$ $\operatorname{Var}\left(X_{2 \tau}-X_{\tau}\right)$ in Proposition 1 is satisfied for all $\tau \in \mathbb{N}$.

The above proposition shows that the change probabilities (and thus the scaledependent Hurst exponents) of a Gaussian process are, under some stationarity conditions, related to its variance, which in turn is related to the covariance, as can be seen from (4). In the following the classical Hurst exponent will be discussed, which is based on the autocorrelation and therefore also on the covariance of the process. In particular, a relationship between scale-dependent Hurst exponents and their classical counterpart will be established. 
For a stochastic process $\left(X_{t}\right)_{t \in[0, \infty]}$ satisfying (A1), (A2), (A3) and

$$
\left.0<\operatorname{Var}\left(X_{t}\right)<\infty \quad \text { for all } t \in\right] 0, \infty[
$$

consider the autocorrelation function $c:[0, \infty[\rightarrow \mathbb{R}$ of the increment process $\left(X_{t+1}-X_{t}\right)_{t \in[0, \infty[\text { which is defined by }}$

$$
c(\tau):=\operatorname{Cov}\left(X_{\tau+1}-X_{\tau}, X_{1}\right) / \operatorname{Var}\left(X_{1}\right),
$$

for $\tau \in\left[0, \infty\right.$ [. (In case $\operatorname{Var}\left(X_{1}\right)=0$, not relevant at this point, one defines $c(\tau)=0$.)

Recall that for fBm with Hurst exponent $H$ it holds that

$$
c(\tau)=\frac{1}{2}\left(|\tau+1|^{2 H}-2|\tau|^{2 H}+|\tau-1|^{2 H}\right)
$$

for all $\tau \in[0, \infty[$ and

$$
\lim _{\tau \rightarrow \infty} \frac{c(\tau)}{\tau^{2 H-2}}=H(2 H-1)
$$

(e.g., Gneiting and Schlather (2004)). The latter formula describes the asymptotic behavior of $c$ for large $\tau$ and gives rise to a general definition of the Hurst exponent $H$ of $\left(X_{t}\right)_{t \in \mathbb{N}_{0}}$ as the number

$$
H:=1+\frac{1}{2} \lim _{\tau \rightarrow \infty} \frac{\log (|c(\tau)|)}{\log (\tau)}
$$

provided this limit exists (see, e.g., Gneiting and Schlather (2004)). To see that this definition is consistent with the role of the Hurst parameter for $\mathrm{fBm}$, assume that the limit

$$
\lim _{\tau \rightarrow \infty} \frac{c(\tau)}{\tau^{2 H-2}} \text { exists for some } H \text { and differs from } 0
$$

Then $H$ is necessarily the Hurst exponent of $\left(X_{t}\right)_{t \in[0, \infty[}$ as defined by (5), which is easily deduced from (A5), see Lemma 3 in the Appendix. Moreover, if $H \geq 0.5$, then the limit in (A5) is necessarily positive, see Lemma 2 in the Appendix. (The last assumption in (A5) implies in particular that the quotient $c(\tau) / \tau^{2 H-2}$ is bounded away from zero for large $\tau$ : there exist some constant $C, \tau_{0}>0$ such that either $c(\tau) \tau^{2-2 H}>C$ for all $\tau \geq \tau_{0}$ or $-c(\tau) \tau^{2-2 H}>C$ for all $\tau \geq \tau_{0}$. This means that $c$ will not change its sign for $\tau \geq \tau_{0}$.)

In the sequel, all considerations will be restricted to Gaussian processes $\left(X_{t}\right)_{t \in[0, \infty[}$ satisfying the conditions (A1)-(A5). By the above, this implies in particular that the Hurst exponent of $\left(X_{t}\right)$ exists. Note that if $\left(X_{t}\right)$ is not a fBm, then the values of $p(\tau)$ may vary with $\tau \in] 0, \infty[$, and therefore $H(\tau)=h(p(\tau))$ can be different from the Hurst exponent for some $\tau \in] 0, \infty[$. However, the above conditions ensure that the Hurst exponent $H(\tau)$ of delay $\tau$ converges to the Hurst exponent $H$ as $\tau \rightarrow \infty$. 
Theorem 1 Let $\left(X_{t}\right)_{t_{\in}[0, \infty[}$ be a Gaussian process satisfying the conditions (A1)(A4). Assume that condition (A5) is satisfied for some constant $H$ such that $0.5 \leq$ $H<1$. Then the Hurst exponent (as defined in (5)) exists and equals $H$, and

$$
\liminf _{\tau \rightarrow \infty} H(\tau) \leq H \leq \limsup _{\tau \rightarrow \infty} H(\tau)
$$

holds true. Thus, if the limit

$$
\lim _{\tau \rightarrow \infty} H(\tau)
$$

exists, then it necessarily equals $H$.

Theorem 1 establishes an asymptotic relation between change probabilities and the Hurst exponent, which is the best one can hope for in a situation when there is no self-affinity present. As the assumptions in Theorem 1 are rather mild, one can expect them to be satisfied by many stochastic processes. Examples covered by Theorem 1 that are not $\mathrm{fBm}$ include those provided by the processes discussed in Gneiting and Schlather (2004): if $\left(X_{t}\right)$ is a Gaussian process satisfying (A1) such that its increment process is stationary and has a correlation function from the Cauchy class (or any of the other classes discussed in Gneiting and Schlather (2004)), then the conditions (A2)-(A5) are satisfied. The proof of Theorem 1 can be found in the Appendix.

Note that for data of rock surfaces, the Hurst exponent is typically larger than 0.5 (see, e.g., Candela et al. 2012). Therefore, the condition $H \geq 0.5$ is not a serious restriction from the practical viewpoint.

Compared to the classical setting of $\mathrm{fBm}$, the more general class of processes behind Theorem 1 increases flexibility when working with Hurst exponents as a roughness measure. Now roughness is allowed to vary with the delay. Only some stabilization for increasing delays is assumed, allowing one to still consider a single Hurst exponent. This provides a theoretical framework for the case that some stabilization of (estimated) Hurst exponents is observed for real data.

\subsection{Estimating Roughness}

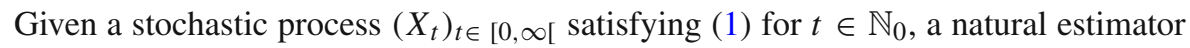
of $p(\tau)$ for some $\tau \in] 0, \infty[$ and $N \in \mathbb{N}$ is given by

$$
\widehat{p}^{(N)}(\tau):=\frac{1}{N} \sum_{t=0}^{N}\left(1_{\left\{X_{t}<X_{t+\tau}, X_{t+\tau} \geq X_{t+2 \tau}\right\}}+1_{\left\{X_{t} \geq X_{t+\tau}, X_{t+\tau}<X_{t+2 \tau}\right\}}\right) .
$$

The following considerations are restricted to the case $\tau \in \mathbb{N}$. Principally, it is enough to start with a process $\left(X_{t}\right)_{t \in \mathbb{N}_{0}}$. Assume that for such a process, the increment process $\left(X_{t+1}-X_{t}\right)_{t \in \mathbb{N}_{0}}$ is a non-degenerated zero-mean stationary Gaussian process. Nondegenerated means that each of the finite distributions of the process is absolutely continuous with respect to the Lebesgue measure. 
If $c(\tau) \rightarrow 0$ as $\tau \rightarrow \infty$, then $\widehat{p}^{(N)}(\tau)$ is a strongly consistent and asymptotically unbiased estimator $(N \rightarrow \infty)$ of $p(\tau)$ for $\tau \in \mathbb{N}$. If, moreover,

$$
|c(\tau)| \cdot \tau^{\beta} \rightarrow 0 \text { as } \tau \rightarrow \infty \text { for some } \beta<0.5,
$$

then the estimator is asymptotically normally distributed as $N \rightarrow \infty$. This was shown in Sinn and Keller (2011b) in the general context of ordinal pattern probabilities for $\tau=1$ using an asymptotic result of Arcones (1994); the generalization to $\tau \neq 1$ is straightforward. The idea goes back to the work of Ho and Sun (1987), where zero-crossings in a process are discussed. Note that a change in the original process corresponds to a zero-crossing in the associated increment process. Note also that (7) is satisfied if condition (A5) holds with $0<H<0.75$.

When passing from $\widehat{p}^{(N)}(\tau)$ to $\widehat{H}^{(N)}(\tau):=h\left(\widehat{p}^{(N)}(\tau)\right)$ and from $p(\tau)$ to $H(\tau)=$ $h(p(\tau))$, all described estimation properties remain true, which follows by applying the delta method (see, e.g., Doob 1935). In particular, for each $\tau \in \mathbb{N}, \widehat{H}^{(N)}(\tau)$ is an estimator of the Hurst exponent $H(\tau)$ with the described properties. It is interesting to note that the estimators $\widehat{p}^{(N)}(\tau)$ and $\widehat{H}^{(N)}(\tau)$, which are based only on the ordinal structure of a time series, show a good performance, and in particular have a low bias (see Sinn and Keller 2011b).

In the situation of Theorem 1 , the Hurst exponent $H$ can still be estimated by $\widehat{H}^{(N)}\left(2^{n}\right)$ with sufficiently large $n$ and $N$.

\section{An Algorithm for Visualizing and Detecting Fracture Roughness Anisotropy}

In this section, an algorithm is described that takes a two-dimensional gray-level image as input and returns an array of estimates of the Hurst exponents $H(\tau)$ for a number of directions $\phi$ and delays $\tau$. The algorithm may be viewed as an application of Theorem 1. In this theorem, the data are modeled by a stochastic process $X_{t}$ with $t \in \mathbb{R}$, while in practice one is dealing with discrete data, i.e., $X_{t}$ with $t \in \mathbb{N}_{0}$. Here it is necessary to determine what $t=1$ should mean, i.e., to fix a minimal spatial scale. Since pixel images are considered here, it makes sense to set the width of one pixel to 1 , which is the smallest meaningful distance between two points in these images. However, when comparing images of different resolutions, the size of a pixel might correspond to different physical distances, and then one has to be careful to compare quantities of physically matching delays.

\subsection{Input}

The input of the algorithm is a two-dimensional matrix $A=\left(a_{i, j}\right) \in \mathbb{R}^{w_{x} \times w_{y}}$ of width $w_{x} \in \mathbb{N}$ and height $w_{y} \in \mathbb{N}$, where the entry $a_{i, j}$ corresponds to the relative height of the two-dimensional rock surface at the position $(i, j)$. This matrix can be represented as a gray-level image, where the brightness of the pixel at position $(i, j)$ is equal to the value of $a_{i, j}$. 


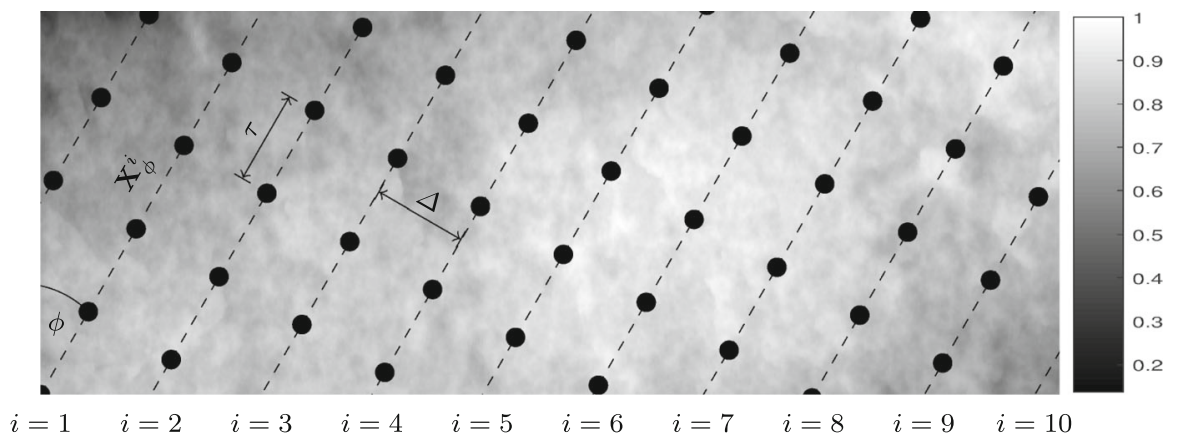

Fig. 2 Exemplary input matrix of a natural sandstone fracture surface. The value of 1 corresponds to the maximum height of the fracture surface; the value zero corresponds to the minimum height. In step 2 of the algorithm, parallel one-dimensional profiles $\mathbf{X}_{\phi}^{i}$ are extracted for a given angle $\phi$

Additional inputs are $n_{\phi} \in \mathbb{N}$, a parameter of the algorithm fixing the number of angular directions $\phi$ for which profile lines are considered, and a vector $\vec{\tau}$ of length $n_{\tau}$ containing the delays $\tau$ at which the one-dimensional profiles will be scanned. The algorithm will choose $n_{\phi}$ angles equally spaced between $0^{\circ}$ and $180^{\circ}$, and the delays contained in $\vec{\tau}$ are positive integers. Optional input is a parameter $\Delta$ which fixes the distance between neighboring parallel profile lines (Fig. 2). The default value of $\Delta$ is 1 . Recall that value 1 for the delay $\tau$ or the parameter $\Delta$ corresponds to a length equal to the width of a pixel.

\subsection{Algorithm}

1. Preparation of the data: A possible two-dimensional linear trend is removed from the input matrix $A$ by performing a linear regression and subtracting the resulting regression plane from $A$.

2. Extraction of one-dimensional profiles: For each of the $n_{\phi}$ angles $\phi$, create onedimensional profiles in direction $\phi$ with delay 1 . To achieve this, consider first a line in the image through the origin in direction $\phi$, i.e., having angle $\phi$ with the vertical axis (see Fig. 2). Take a sequence of equally spaced points on that line which are a pixel width apart from each other.

For each of these points, calculate the height value at the corresponding position using linear interpolation of the given data $A$. Repeat this procedure for all lines intersecting the image that are parallel to the first line and an integer multiple of $\Delta$ apart from it. Each of the one-dimensional profiles extracted in this way will be saved in a vector $\mathbf{x}_{\phi}^{i}=\left(x_{\phi, t}^{i}\right)_{t=0}^{m_{\phi, i}}$ where $i=1,2, \ldots, k_{\phi, \Delta}$. Here, $k_{\phi, \Delta}$ denotes the number of parallel lines intersecting the image for the given $\phi$ and $\Delta$, and $m_{\phi, i}$ +1 is the number of data points in the $i$-th profile.

3. Calculation of change probabilities: For each angle $\phi$ and each delay $\tau$, do the following calculations: For each profile $i \in\left\{1,2, \ldots, k_{\phi, \Delta}\right\}$ with $m_{\phi, i} \geq 2 \tau$, compute the number of changes 


\section{Input data}
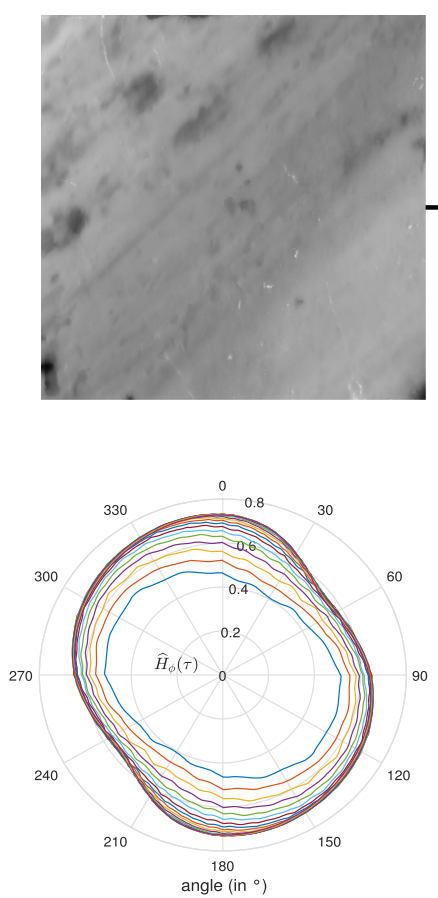

Hurst exponent
One-dimensional profiles

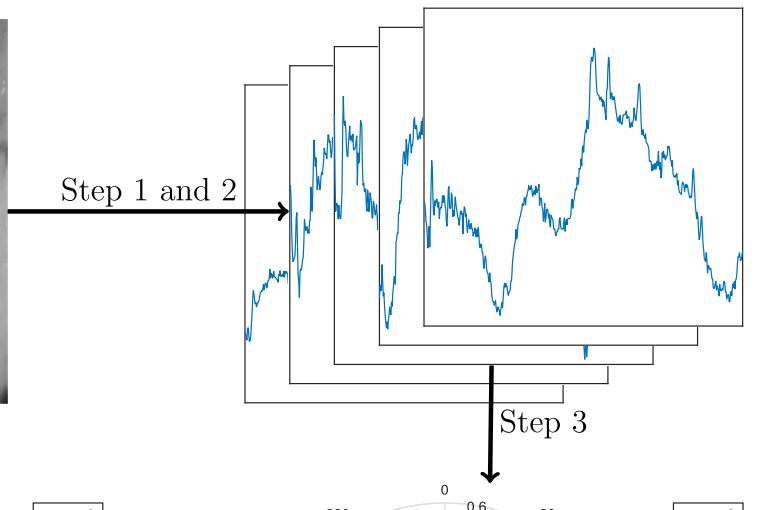

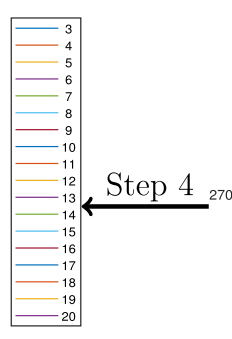

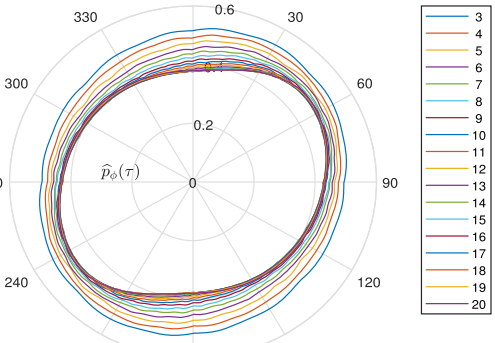

15

180

angle (in ${ }^{\circ}$

Change probabilities

Fig. 3 Diagram illustrating the different steps of the algorithm described in Sect. 3.2. The polar plots that are created in steps 3 and 4, respectively, show the computed change probability and Hurst exponent as a function of the angle $\phi$ for different delays $\tau$ (represented by different colors)

$$
\begin{aligned}
\text { numChanges }_{\phi, \tau, i}:= & \sum_{j=0}^{m_{\phi, i}-2 \tau} \delta\left(x_{\phi, j}^{i}<x_{\phi, j+\tau}^{i} \text { and } x_{\phi, j+\tau}^{i} \geq x_{\phi, j+2 \tau}^{i}\right) \\
& +\delta\left(x_{\phi, j}^{i} \geq x_{\phi, j+\tau}^{i} \text { and } x_{\phi, j+\tau}^{i}<x_{\phi, j+2 \tau}^{i}\right),
\end{aligned}
$$

where $\delta(B)=1$ if the assertion $B$ is true and $\delta(B)=0$ otherwise. (For $m_{\phi, i}<2 \tau$, numChanges $_{\phi, \tau, i}$ is set to 0 .) The estimator $\widehat{p}_{\phi}(\tau)$ for the change probability is then calculated by

$$
\widehat{p}_{\phi}(\tau)=\frac{\sum_{i=1}^{k_{\phi, \Delta}} \text { numChanges }_{\phi, \tau, i}}{\sum_{i=1}^{k_{\phi, \Delta}}\left(m_{\phi, i}-2 \tau+1\right)} .
$$

4. Estimation of roughness: For each angle $\phi$ and each delay $\tau$, estimate the roughness $H_{\phi}(\tau)$ of delay $\tau$ in direction $\phi$ by

$$
\widehat{H}_{\phi}(\tau)=h\left(\widehat{p}_{\phi}(\tau)\right),
$$


where $h$ is defined as in (3).

\subsection{Output}

The output of the algorithm is a $n_{\phi} \times n_{\tau}$ matrix $\widehat{\mathbf{p}}=\left(\widehat{p}_{\phi}(\tau)\right)$ containing the estimates for the change probabilities for each angle-delay pair $(\phi, \tau)$. Optional output is a $n_{\phi} \times n_{\tau}$ matrix $\widehat{\mathbf{H}}=\left(\widehat{H}_{\phi}(\tau)\right)$ containing estimates for the Hurst exponents for each pair $(\phi, \tau)$. Based on this output, the program creates a polar plot of the estimated Hurst exponents $\widehat{H}_{\phi}(\tau)$ as a function of the angle $\phi$ for different delays $\tau$ (see the last step in Fig. 3) and a plot of the estimated Hurst exponents $\widehat{H}_{\phi}(\tau)$ as a function of the delay $\tau$ for different angles $\phi$ (see Fig. 5).

\subsection{Implementation}

The delay $\tau \in \mathbb{N}$ should be chosen to be larger than 2 . If the considered angle $\phi$ is not a multiple of $90^{\circ}$, then the values for the one-dimensional profiles need to be extracted using interpolation. For $\tau \leq 2$, the values of two consecutive points $x_{\phi, t}^{i}$ and $x_{\phi, t+\tau}^{i}$ on the profile are so close together that they partially depend on the same values of the original input data. As a result, the interpolated one-dimensional profile will be smoother than the original rock fracture. Hence, the actual roughness will be underestimated. For angles $\phi$ close to a multiple of $90^{\circ}$, this effect is less prominent.

As an alternative to using linear interpolation for determining intermediate values, one could, for example, use nearest-neighbor interpolation. But experiments have shown that the type of interpolation does not have a significant effect on the outcome.

Additionally, note that the calculations of change probabilities for different angles do not depend on each other and can therefore be processed in parallel. For the source code for this algorithm see Gutjahr (2021).

\subsection{Estimating a Scale-Independent Hurst Exponent}

Given the estimators $\widehat{H}_{\phi}(\tau)$ for the scale-dependent Hurst exponents, it is generally not clear what the best way would be to derive from them a single estimator for the (scale-independent) Hurst exponent in a given direction. Considering such a single estimator would, of course, only be justified in the presence of at least some selfaffinity. The observation of some range of delays, for which the Hurst exponents do not change significantly, may give rise to the hypothesis that the underlying structure is self-affine for this range of delays. It is then reasonable to expect that the Hurst exponents for such delays best describe the given data. In this situation, the median value of the estimators $\widehat{H}_{\phi}(\tau)$ of the scale-dependent Hurst exponents, where $\tau$ varies in the specified range of delays, may be a good estimator for the scale-independent Hurst exponent $H_{\phi}$ (for profiles in direction $\phi$ ). The median as an estimator of the Hurst exponent has the additional advantage of being insensitive to outliers, as can be seen in Fig. 7. The outcome of this method when applied to two different natural fracture surfaces is depicted in Fig. 8. 
The median is taken over the values $\widehat{H}_{\phi}(\tau)$ for $\tau \in\left\{3,4, \ldots, \tau_{\max }\right\}$, where one needs to specify how to choose $\tau_{\max }$. As explained in Sect. 3.4, the value of $\widehat{H}_{\phi}(\tau)$ for $\tau \in\{1,2\}$ is not taken into account.

Clearly, $\tau_{\max }$ should depend on the size of the image and is determined by

$$
\tau_{\max }=\left\lceil\sqrt[4]{w_{X} \cdot w_{Y}}\right\rceil
$$

where $w_{X}$ and $w_{Y}$ are the widths of the image in the $X$ - and $Y$-direction measured in pixels. It is motivated by the need to find a compromise between the pattern length $(2 \tau+1)$ and the number of patterns. Increasing the length decreases the number and vice versa. Choosing for a profile of length $L$ a delay of order $\sqrt{L}$ results roughly in a number of $\sqrt{L}$ non-overlapping patterns. Here, non-overlapping means that the intervals spanned by the pattern locations are disjoint for different patterns. Since in a rectangular image the length $L$ of the profiles changes with the direction, the geometric mean of the profile lengths in the $X$ - and $Y$-direction is chosen as a compromise, resulting in the value $\tau_{\max }=\left\lceil\sqrt{\sqrt{w_{X} \cdot w_{Y}}}\right\rceil$.

\section{Application to Natural Fracture Surfaces}

In this section, the algorithm introduced above is applied to gray-level images of two natural fractures, namely a tensile and a shear fracture. The analyzed tensile fracture is a bedding-parallel joint in a carbonate-cemented aeolian sandstone, which opened up within a block specimen sampled from the Schwentesius outcrop in northern Germany (Fischer et al. 2007; Heidsiek et al. 2020). Since it can be described as an openingmode fracture, the surface was not exposed to shear stress. A handheld laser scanner was used to produce a three-dimensional image of the rough fracture surface (Fig. 4b). It is represented by a point cloud, corresponding to a set of data points where the spatial position of each point is uniquely defined by $X, Y$, and $Z$ coordinates. The average point spacing is $0.157 \mathrm{~mm}$. Further information on the specifications of the laser scanner, as well as a photo of the bedding-parallel tensile fracture, is provided by Hale et al. (2020). The fracture surface does not show a discernible roughness anisotropy except for a barite vein, which cuts through the bedding plane and is noticeable as an elevated ridge-like structure. To make sure that this vein does not skew the result when estimating the roughness of this fracture, the right part of this surface, beginning at $350 \mathrm{~mm}$, is cut off before applying any methods to it (Fig. 2).

Furthermore, a fresh fault slip surface in limestone was used as additional input data for the subsequent roughness analysis (Fig. 4a). It is part of the Bolu outcrop in Turkey, which is appendant to the North Anatolian Fault zone (Candela et al. 2012; Renard et al. 2013). The raw data can be found at ISTerre (2021). In contrast to the tensile fracture (Fig. 4b), the sampled fault surface was exposed to shear stress and has experienced a minimum movement of approximately $20 \mathrm{~m}$ (Candela et al. 2012). The fault slip surface shows characteristic features such as elongated lenses and linear striae which are also evident from Fig. 3 in Candela et al. (2012) and Fig. 1 in Renard et al. (2013). For fault slip surfaces, a distinct roughness anisotropy was observed on various length scales, evident from deviating Hurst exponents or fractal dimensions 


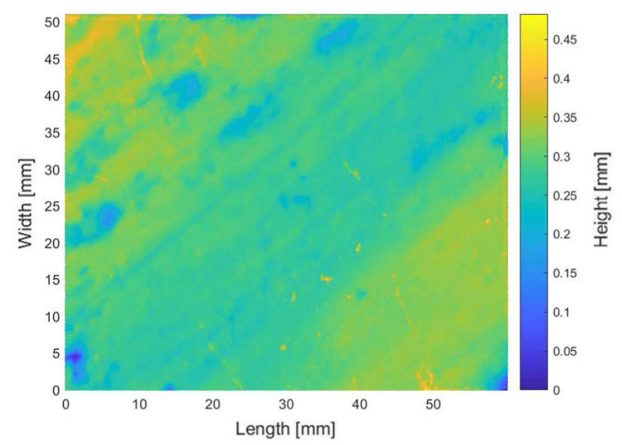

(a)

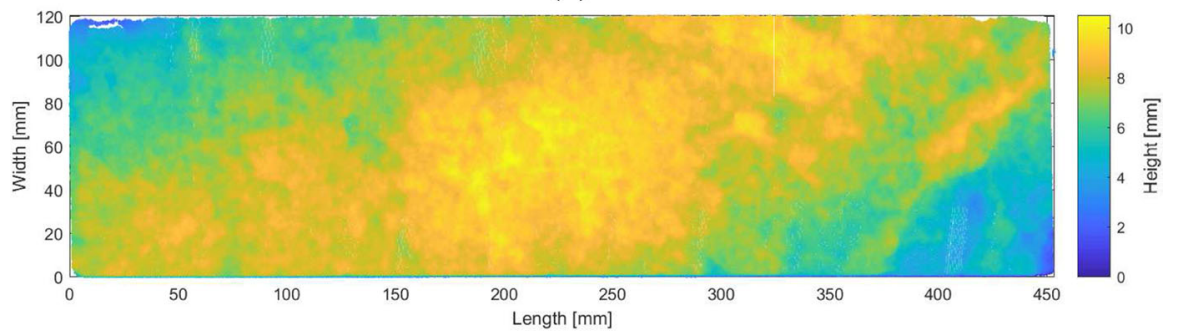

(b)

Fig. 4 Point cloud data of a a natural shear plane from a limestone outcrop ("Bolu-1", Candela et al. 2012) and b a natural bedding-parallel tensile fracture in sandstone. The $X$ - and $Y$-axis correspond to the length and width of the captured surface segment, respectively; the $Z$-coordinate, indicated by the color bars, specifies the surface height (all units are in $\mathrm{mm}$ ). For the tensile fracture, the $Z$-coordinate (surface height) varies between 0 and $10.53 \mathrm{~mm}$ (b), while the surface height variation of the fault plane segment (a) is below $500 \mu \mathrm{m}$

parallel and perpendicular to the slip direction (e.g., Candela et al. 2012; Renard et al. 2006; Lee and Bruhn 1996). The point cloud was produced by a laboratory laser profilometer (Fig. 4a) and shows a regular point spacing of $20 \mu \mathrm{m}$ (Candela et al. 2012).

Based on the three-dimensional point cloud data of the fracture surfaces, twodimensional gray-level images are created which are required as input data for the algorithm presented in Sect. 3.2. First, a surface is fitted to the scattered point data by nearest-neighbor interpolation employing the standard MATLAB routine. In this context, a mesh grid has to be specified for evaluating the created interpolant at designated locations. The resolution of the mesh grid should ideally match the resolution or the average point spacing of the point cloud in order to prevent a loss of roughness information. Here, the mesh resolution was set to $0.15 \mathrm{~mm}$ for the tensile fracture and to $0.02 \mathrm{~mm}$ for the fault slip surface. If necessary, the resulting matrix is cropped to remove less dense areas at the edges of the original point cloud. To export the matrix in binary pgm format, the surface height information (i.e., matrix values) is rescaled to fit into the interval $[0,255]$, where 0 corresponds to the minimum and 255 to the maximum surface height of the fracture surface. 


\subsection{Dependence on the Delay}

Figure 5 visualizes how the estimates $\widehat{H}_{\phi}(\tau)$ of the Hurst exponent change with the delay $\tau$ for fixed angles $\phi$. Figure 5a indicates that, independent of the angle $\phi$, the values of $\widehat{H}_{\phi}(\tau)$ increase with $\tau$ until a maximum is reached at around $\tau=19$, and then decrease until around $\tau=80$. In Fig. 5b, the values of $\widehat{H}_{\phi}(\tau)$ decrease with $\tau$ until a minimum is reached near $\tau=40$. The values then increase slightly, and the average over all angles stabilizes near 0.4 .

For comparison, scale-dependent Hurst exponents are also estimated for simulations of fBm, using the same estimator based on change probabilities as for the rock profiles. The results are plotted as a red line in Fig. 5. The line is derived from 100 stochastically independent simulations of $\mathrm{fBm}$, whose length equals the geometric mean of width and height (in pixels) of the corresponding rock surface. The $\tau$-dependent Hurst exponents (see Eq. (9)) are computed for each simulation and then the average is taken. The Hurst exponent for the simulations was chosen as 0.46 for the shear plane (left) and 0.51 for the tensile fracture (right) so that they roughly correspond to the mean of the estimated Hurst exponents for the individual rock surfaces. Since the simulations of $\mathrm{fBm}$ are one-dimensional, this estimation does not depend on the angle $\phi$. The observations indicate that for both fracture types the scale-dependent Hurst exponents indeed depend to some extent on the delay.

\subsection{Comparison to a Standard Method}

Figure 6 compares the results of the estimator $\widehat{H}_{\phi}(\tau)$ for $\tau=3$, 5, and 25 with the results provided by a standard method. The standard estimator $\widehat{H}_{\text {wavelet }}$ is based on a method which is already implemented in the MATLAB Wavelet Toolbox. This method estimates the variances of wavelets at different detail levels and performs a regression of those variances versus the detail level in a log-log plot (see, e.g., Flandrin 1992; Abry et al. 2000 for details). Technically, the wavelet-based estimator provides an estimate for the Hurst exponent only if the underlying process is a $\mathrm{fBm}$. For more general Gaussian processes, one can still calculate the value of $\widehat{H}_{\text {wavelet }}$, and it is reasonable to assume that it still represents some measure of roughness, but it is not necessarily equal to the Hurst exponent as defined in (5). It might be possible to interpret $\widehat{H}_{\text {wavelet }}$ roughly as some weighted average of the scale-dependent Hurst exponents over the available scales, but this is not obvious. Certainly its computation involves information from many delays.

Figure $6 \mathrm{a}$ shows that the estimates of the Hurst exponent based on $\widehat{H}_{\phi}(3)$ and $\widehat{H}_{\text {wavelet }}$ are very close to each other for all angles $\phi$, whereas the one based on $\widehat{H}_{\phi}(25)$ yields generally larger values than these two. Moreover, the values of $\widehat{H}_{\phi}(25)$ vary more smoothly with $\phi$ than the values of $\widehat{H}_{\phi}(3)$ and $\widehat{H}_{\text {wavelet. All curves have in }}$ common that they attain their minimum value for angles around $50^{\circ}$ and a maximum near $140^{\circ}$.

In Fig. $6 \mathrm{~b}$, the values of $\widehat{H}_{\text {wavelet }}$ are close to those of $\widehat{H}_{\phi}(5)$ for most $\phi$. The curves for $\widehat{H}_{\phi}(3), \widehat{H}_{\phi}(5)$, and $\widehat{H}_{\text {wavelet }}$ are more circular in shape than those obtained for the other fracture in Fig. 6a. 

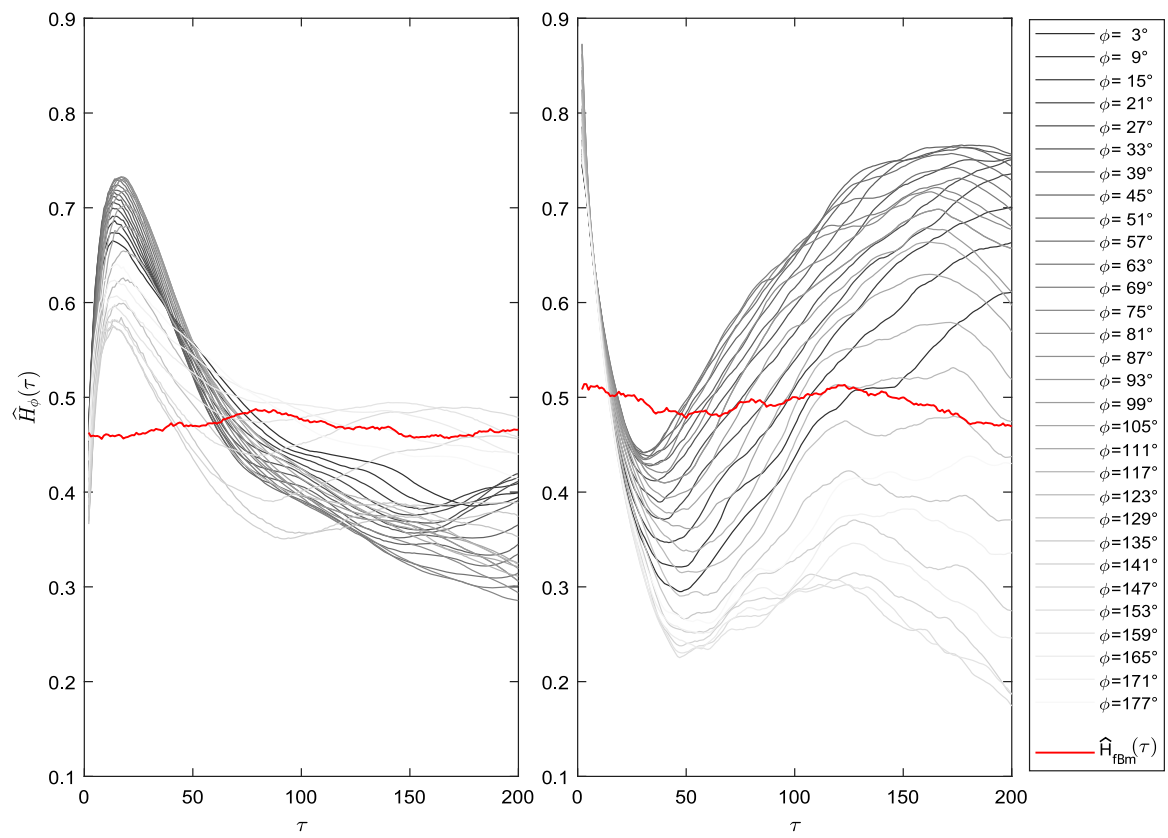

Fig. 5 Estimates $\widehat{H}_{\phi}(\tau)$ of the Hurst exponent (vertical axis) are plotted versus the delay $\tau$ (horizontal axis) for different angles $\phi$ for (left) the shear plane from the Bolu outcrop (Fig. 4a) and (right) the tensile fracture from the Schwentesius outcrop (Fig. 4b). Each of the different gray lines corresponds to one of 30 angles equally spaced between $0^{\circ}$ and $180^{\circ}$. The red line $\widehat{H}_{\mathrm{fBm}}(\tau)$ shows estimates of the $\tau$-dependent Hurst exponents for $\mathrm{fBm}$ based on 100 simulations of $\mathrm{fBm}$

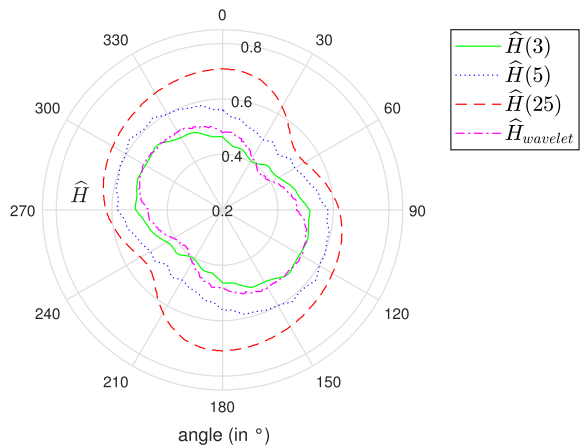

(a)

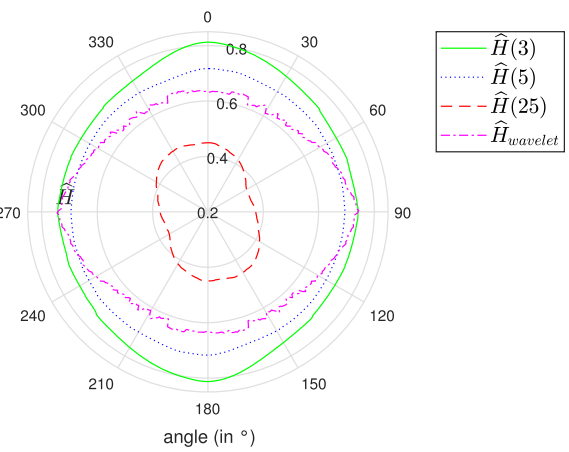

(b)

Fig. 6 Radial plots of estimated Hurst exponents as functions of the angle $\phi$ for a the shear plane (Fig. 4a) and $\mathbf{b}$ the tensile fracture (Fig. 4b). The solid, dotted, and dashed lines correspond to the estimates based on $\widehat{H}_{\phi}(\tau)$ for the delays $\tau=3,5$, and 25, respectively. The dash-dotted line shows the estimated Hurst exponent $\widehat{H}_{\text {wavelet }}$ using the wavelet-based method implemented in MATLAB 


\subsection{Noise Effects}

Figure 7 shows the effect of noise on the estimators for the Hurst exponent. To simulate this effect, white noise with mean zero and variance $\sigma^{2}$ was added to each pixel of the image. The Hurst exponent was then estimated for the noisy image using, on the one hand, the method implemented in MATLAB and on the other hand the new proposed estimators $\widehat{H}_{\phi}(\tau)$ for different values of $\tau$. The squares of the differences between the resulting estimates $\widehat{H}_{\phi}^{\sigma}$ and the estimates $\widehat{H}_{\phi}$ for the undisturbed image were summed over all considered angles $\phi$. The error is then defined as the square root of this sum divided by the number $n_{\phi}$ of different angles

$$
\text { error }=\frac{1}{n_{\phi}} \sqrt{\sum_{\phi}\left(\widehat{H}_{\phi}^{\sigma}-\widehat{H}_{\phi}\right)^{2}}
$$

As a reference for the scale of the image values, the square root of the squared average deviation of the pixel values from the mean image value is considered, which is given by

$$
\sigma_{\mathrm{img}}=\sqrt{\frac{1}{w_{x} \cdot w_{y}-1} \sum_{i=1}^{w_{x}} \sum_{j=1}^{w_{y}}\left(a_{i, j}-\frac{1}{w_{x} \cdot w_{y}} \sum_{i^{\prime}=1}^{w_{x}} \sum_{j^{\prime}=1}^{w_{y}} a_{i^{\prime}, j^{\prime}}\right)^{2}},
$$

where $\left(a_{i, j}\right)_{i, j=1}^{w_{x}, w_{y}}$ are the pixel values of the image.

In Fig. 7, the effect of noise on different estimators is studied for the shear plane (Fig. 4a). For this purpose, white noise with standard deviation $\sigma$ was added to the surface for different values of $\sigma$ ranging from $10^{-1}$ to $10^{-4}$. For comparison, the standard deviation over the image as defined in (12) is approximately equal to $\sigma_{\text {img }} \approx$ 0.064 . For the computation of the error, the profiles in $n_{\phi}=20$ different directions $\phi$ (chosen equally spaced between 0 and $\pi$ ) are taken into account.

\section{Discussion}

\subsection{Computational Advantages}

From the computational point of view, the estimator $\widehat{H}_{\phi}(\tau)$ has some advantages compared to alternative methods. Firstly, no linear regression of some logarithmic property is needed to determine $\widehat{H}_{\phi}(\tau)$. In case the data can be modeled by $\mathrm{fBm}$, the value of $\widehat{H}_{\phi}(\tau)$ for just one delay $\tau$ is a sufficient estimator for the Hurst exponent. In contrast, for other variance-based methods, some property of the data needs to be estimated over many different scales $\tau$, and the Hurst exponent is then obtained as the slope of some linear function of (the logarithm of) $\tau$ determined by this property. Thus, repeated calculations for multiple delays are necessary in this case.

Further, the calculations needed to determine $\widehat{p}_{\phi}(\tau)$ (and thus $\widehat{H}_{\phi}(\tau)$ ) via the number of changes as given by (8) are mathematically very elementary. One only needs to 


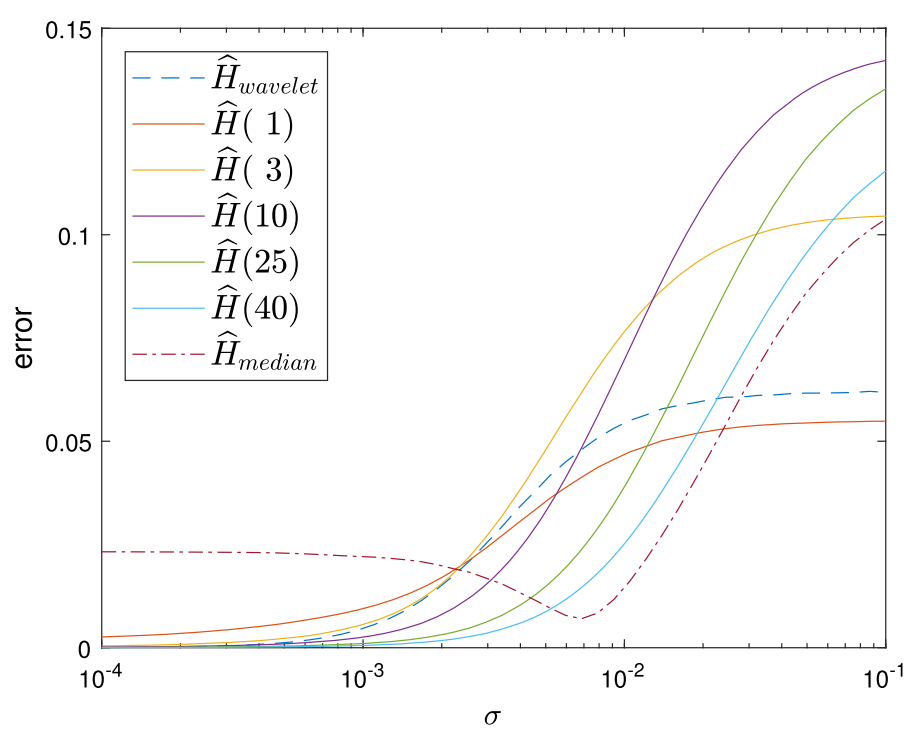

Fig. 7 The effect of adding white noise of standard deviation $\sigma$ to the shear plane (Fig. 4a) is presented for different roughness estimators. The plots show the error (as defined in (11) with $n_{\phi}=20$ equally spaced angles) as a function of $\sigma$. The dashed line corresponds to the estimator based on wavelets mentioned in Sect. 4.2, the dash-dotted line to the median estimator proposed in Sect. 4.1, and the solid lines to the estimators based on change probabilities for different delays

compare pairs of values, apply some Boolean operations, and then count the number of true results. Essentially no costly multiplications are necessary. Hence, the necessary calculations can be done very quickly and, moreover, they can easily be parallelized.

\subsection{Detection of Anisotropy}

Comparing Fig. 6a and b, one can observe that the closed lines in the polar plots describing the angle-dependent roughness are much more circular in Fig. $6 \mathrm{~b}$ than in Fig. 6a. The same can be observed when comparing the median roughness in Fig. 8b with the one in Fig. 8a. Therefore, one can conclude that the tensile fracture exhibits less roughness anisotropy than the shear plane. This is in line with the fact that the tensile fracture shows no visible anisotropy, which can be expected due to the formation mechanism.

In contrast, the existing roughness anisotropy of the shear plane was detected with the methods introduced here.

\subsection{Dependence on the Delay}

Figure 5 shows that the value of the $\widehat{H}_{\phi}(\tau)$ is not constant over all delays $\tau$. One could ask whether this is merely a statistical phenomenon caused by fluctuating estimates. However, the clear monotonic behavior of the estimates as a function of $\tau$ contradicts 


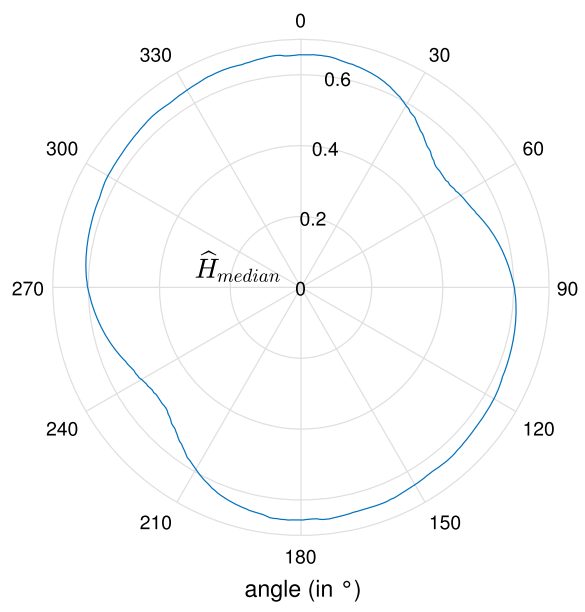

(a) Shear fracture $\left(\tau_{\max }=53\right)$

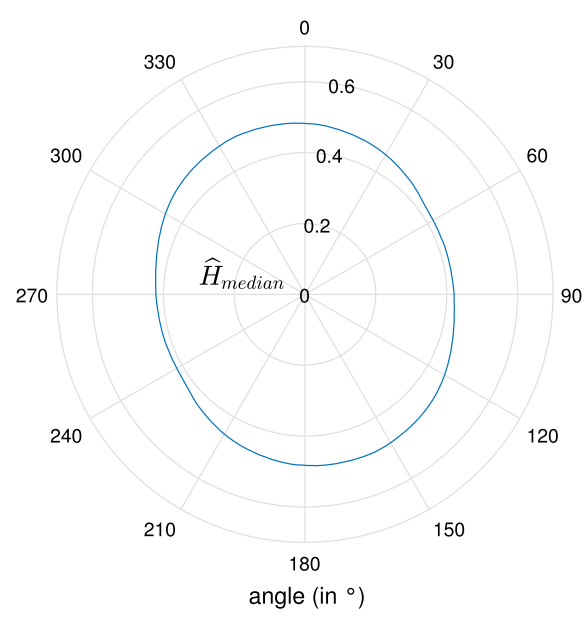

(b) Tensile fracture $\left(\tau_{\max }=37\right)$

Fig. 8 Polar plots showing for the two natural fracture surfaces (Fig. 4) the median Hurst exponent on the radial axis in dependence of the angle $\phi$. It was calculated by taking for each angle $\phi$ the median value of the change probabilities for integer delays $\tau$ between 3 and $\tau_{\max }$ and then applying formula (3) to this median. $\tau_{\max }$ was determined according to Eq. (10)

such a hypothesis. Comparing the fluctuations of $\widehat{H}_{\phi}(\tau)$ to the fluctuations of corresponding estimates $\widehat{H}_{\mathrm{fBm}}(\tau)$ of $\mathrm{fBm}$ (see the red line in Fig. 5) further contradicts this hypothesis. Already a rough visual comparison shows that the behavior of $\widehat{H}_{\mathrm{fBm}}(\tau)$ and $\widehat{H}_{\phi}(\tau)$ differs significantly. Additionally, the relatively small fluctuations of the red line confirm that for $\mathrm{fBm}$, the $\tau$-dependent Hurst exponents do not depend on the delay $\tau$, as explained in Sect. 2.2. The dependence of the estimated Hurst exponents $\widehat{H}_{\phi}(\tau)$ on the delay indicates that modeling of the considered data by fBm only makes sense to a limited extent.

The delays $\tau=19$ in Fig. $5 \mathrm{a}$ and $\tau=40$ in Fig. 5 b, for which $\widehat{H}_{\phi}(\tau)$ is maximal or minimal, respectively, might correspond to the length of some characteristic structure on the fracture surface. The clear maximum visible in Fig. 5 could, potentially, be used to identify such characteristic lengths.

For larger delays $\tau$, the value of $\widehat{H}_{\phi}(\tau)$ seems to fluctuate less and stabilize around some constant value depending on the angle $\phi$. This observation is consistent with the theoretical results in Sect. 2.3.

Additionally, note that $\widehat{H}_{\phi}(\tau)$ is, on average, tending towards values around 0.5 for large $\tau$ in Fig. 5a and b. Recall that data with independent increments can be modeled by a fBm with Hurst exponent $H=0.5$. The above observation could therefore be explained by the fact that the stochastic dependence between points on the fracture surface, which are separated by a large distance $\tau$, decreases faster than predicted by the mathematical model for the fracture surface. 


\subsection{Noise Effects}

Figure 7 shows that the larger the sampling rate $\tau$, the less the estimator $\widehat{H}_{\phi}(\tau)$ is affected by noise. This is not surprising. Since the difference between values $x_{t}$ and $x_{t+\tau}$ is typically smaller on average the smaller the $\tau$, small random perturbations are more likely to cause a change in the pattern of some $\left(x_{t}, x_{t+\tau}, x_{t+2 \tau}\right)$ for smaller $\tau$. Furthermore, one can see that the estimator $\widehat{H}_{\phi}(\tau)$ is less affected by noise than the wavelet-based estimator $\widehat{H}_{\text {wavelet }}$, provided that the strength of the noise is not too large. The reason for this is that small random perturbations of given data $\left(x_{t}\right)_{t=1}^{n}$ will significantly affect the estimator $\widehat{H}_{\phi}(\tau)$ only if the randomness changes the ordering of $x_{t}, x_{t+\tau}, x_{t+2 \tau}$ for sufficiently many $t$.

\subsection{Comparison to Standard Method}

In Fig. 6, one can see that for specific sampling rates $\tau$, the estimator $\widehat{H}_{\phi}(\tau)$ yields results that are comparable to those with standard methods. Figure 6a shows that, even though the value of $\widehat{H}_{\phi}(\tau)$ is different for larger delays $\tau$, the angles that yield the minimal and maximal Hurst exponent remain unchanged. This indicates that the structure of the rock surface that is causing the anisotropy is invariant over a specific range of scales.

One can additionally observe the greater smoothness of $\widehat{H}_{\phi}(25)$ compared to $\widehat{H}_{\phi}(3)$, which is in line with the effects described in Sect. 5.4. For the same reason, the angle at which the minimum of $\widehat{H}_{\phi}(25)$ over all angles is found, is more pronounced. Hence, the delay $\tau$ should not be chosen too small in general.

For the exemplary fractures considered here, $\tau=5$ is considered reasonable. Since it is difficult to know in advance which value for $\tau$ is a good choice, calculating the median fracture roughness over many delays $\tau$ as shown in Fig. 8 is appropriate in these cases.

\section{Conclusions}

The proposed concept of scale-dependent Hurst exponents based on change probabilities provides scale- and direction-dependent roughness information for rough surfaces beyond the summary information provided by classical (single-valued) roughness measures. Aside from some stationarity, the new method does not require any additional assumption on the studied surface or its directional profiles such as self-affinity or scale-invariance and may therefore be applied in situations when, strictly speaking, existing methods are not applicable because they are based on such assumptions.

In the presence of self-affinity, there is a direct relation to the classical Hurst exponent. As is discussed in Theorem 1, an asymptotic relation between scale-dependent and classical Hurst exponents can even be established for a much wider class of processes than just $\mathrm{fBm}$. This suggests the use of change probabilities (if not as a new independent roughness measure) as a means of estimating the classical Hurst exponent in such situations. 
It was demonstrated that estimators for roughness based on change probabilities yield results similar to those with alternative methods, while having computational advantages and being less sensitive to noise.

On the practical side, by exploring two natural fracture surfaces, it was illustrated how the methods proposed here can be used to quantify fracture roughness and to identify roughness anisotropy. In particular, these methods allow one to investigate how fracture roughness varies across different scales. This is useful, for example, for checking the hypothesis of self-affinity.

In the self-affine case, all estimated scale-dependent Hurst exponents defined on the basis of change probabilities are expected to be close to the true Hurst exponent of the structure. Otherwise, in the absence of self-affinity or milder adequate premises, estimators of a possibly undefined Hurst exponent can yield diverse values that are difficult to interpret. This may even result in conflicting statements about the direction of maximum roughness for anisotropic rock surfaces (i.e., shear or fault surfaces). In this case, the use of the scale-dependent Hurst exponents could possibly help to find anisotropies, while their detection by only one (averaging) quantifier is impossible.

The proposed method may also be useful for extracting additional scale-dependent information about the structure of a fracture surface such as the characteristic size of building blocks of a material.

Funding Open Access funding enabled and organized by Projekt DEAL. We gratefully acknowledge funding by the KIT center MathSEE, seed project "Surface roughness and anisotropy of natural rock joints." Financial support by the Federal Ministry of Education and Research (03G0871D) as part of the project ResKin (Reaction kinetics in reservoir rocks) is also gratefully acknowledged.

Availability of data and material Not applicable.

\section{Declarations}

Conflict of interest The authors declare that they have no conflict of interest.

Code availability The produced MATLAB code is freely available, see Gutjahr (2021).

Open Access This article is licensed under a Creative Commons Attribution 4.0 International License, which permits use, sharing, adaptation, distribution and reproduction in any medium or format, as long as you give appropriate credit to the original author(s) and the source, provide a link to the Creative Commons licence, and indicate if changes were made. The images or other third party material in this article are included in the article's Creative Commons licence, unless indicated otherwise in a credit line to the material. If material is not included in the article's Creative Commons licence and your intended use is not permitted by statutory regulation or exceeds the permitted use, you will need to obtain permission directly from the copyright holder. To view a copy of this licence, visit http://creativecommons.org/licenses/ by/4.0\%.

\section{Appendix: Proof of Theorem 1}

For simplicity, it is assumed throughout in the sequel (and without further mention) that $\left(X_{t}\right)_{t \in[0, \infty[}$ is a Gaussian stochastic process satisfying conditions (A1)-(A4). In some of the statements below, these assumptions could be weakened insignificantly, 
but this is not relevant for proving Theorem 1. Further assumptions will be mentioned explicitly where they are needed.

In the following, the lower and upper Hurst exponents of $\left(X_{t}\right)_{t \in[0, \infty[}$ are defined by

$$
\begin{aligned}
& \underline{H}:=\inf \left\{s \in \mathbb{R}: \liminf _{\tau \rightarrow \infty} \frac{|c(\tau)|}{\tau^{2 s-2}}=0\right\} \text { and } \\
& \bar{H}:=\inf \left\{s \in \mathbb{R}: \limsup _{\tau \rightarrow \infty} \frac{|c(\tau)|}{\tau^{2 s-2}}=0\right\},
\end{aligned}
$$

respectively. In general, the (upper) Hurst exponent provides an upper bound for the asymptotic behavior of the variance $\operatorname{Var}\left(X_{\tau}\right)$, and an assumption like the existence of the limit in (A5) implies that the Hurst exponent determines the asymptotic behavior of $\operatorname{Var}\left(X_{\tau}\right)$ completely. This is a consequence of the fact that $\operatorname{Var}\left(X_{\tau}\right)$ can be written in terms of the autocorrelation function $c$. For any $n \in \mathbb{N}$, one has

$$
\begin{aligned}
\operatorname{Var}\left(X_{n}\right) & =\operatorname{Cov}\left(\sum_{i=0}^{n-1}\left(X_{i+1}-X_{i}\right), \sum_{j=0}^{n-1}\left(X_{j+1}-X_{j}\right)\right) \\
& =\sum_{i=0}^{n-1} \sum_{j=0}^{n-1} \operatorname{Cov}\left(X_{i+1}-X_{i}, X_{j+1}-X_{j}\right) \\
& =\operatorname{Var}\left(X_{1}\right)\left(n c(0)+2 \sum_{k=1}^{n-1}(n-k) c(k)\right) \\
& =\operatorname{Var}\left(X_{1}\right)\left(n+2 \sum_{k=1}^{n-1}(n-k) c(k)\right) .
\end{aligned}
$$

Although the values of $\operatorname{Var}\left(X_{\tau}\right)$ are of interest for any $\tau \in \mathbb{R}$ with $\tau>0$ [see, e.g., (2)], the above relation (14) for integer delays $n \in \mathbb{N}$ will be essential for proving Theorem 1.

The following observation will be useful in the sequel.

Lemma 1 Let $\beta \geq-1, \tau_{0} \in \mathbb{N}$ and $C>0$. Then there exists some $\tau_{1}>\tau_{0}$ such that, for any $n \geq \tau_{1}$,

$$
C \cdot n<\sum_{k=\tau_{0}}^{n-1}(n-k) k^{\beta}
$$

Moreover,

$$
\lim _{n \rightarrow \infty} \frac{\log \left(\sum_{k=\tau_{0}}^{n-1}(n-k) k^{\beta}\right)}{\log (n)}=\beta+2 .
$$


Proof To verify inequality (15) for $n$ large enough, it is shown that $1 / n$ times the right-hand side tends to $+\infty$, as $n \rightarrow \infty$. For $\beta>-1$, it holds that

$$
\begin{aligned}
\frac{1}{n} \sum_{k=\tau_{0}}^{n-1}(n-k) k^{\beta} & =\sum_{k=\tau_{0}}^{n-1} k^{\beta}-\frac{1}{n} \sum_{k=\tau_{0}}^{n-1} k^{\beta+1} \geq \int_{\tau_{0}}^{n-1} k^{\beta} \mathrm{d} k-\frac{1}{n} \int_{\tau_{0}}^{n} k^{\beta+1} \mathrm{~d} k \\
& =\frac{(n-1)^{\beta+1}-\tau_{0}^{\beta+1}}{\beta+1}-\frac{n^{\beta+1}-n^{-1} \tau_{0}^{\beta+2}}{\beta+2} \\
& =\frac{(\beta+2)\left(\frac{n-1}{n}\right)^{\beta+1}-(\beta+1)}{(\beta+1)(\beta+2)} n^{\beta+1}-\frac{\tau_{0}^{\beta+1}}{\beta+1}+\frac{1}{n} \frac{\tau_{0}^{\beta+2}}{\beta+2}
\end{aligned}
$$

The first term in this last expression tends to $+\infty$, as $n \rightarrow \infty$, while the other two summands stay bounded. This shows that the right-hand side of (15) grows faster than $n$ as $n \rightarrow \infty$ such that the inequality (15) must be valid for $n$ large enough. For $\beta=-1$, it holds that $\frac{1}{n} \sum_{k=\tau_{0}}^{n-1}(n-k) k^{\beta}=\sum_{k=\tau_{0}}^{n-1} k^{-1}-1+\frac{\tau_{0}}{n} \rightarrow+\infty$, as $n \rightarrow \infty$. Thus, inequality (15) is also valid in this case for large enough $n$. Recalling that the harmonic series grows like $\log (n)$, it is easily seen that $\lim _{n \rightarrow \infty} \log \left(\sum_{k=\tau_{0}}^{n-1}(n-k) k^{\beta}\right) / \log (n)=\lim _{n \rightarrow \infty} \log (n \log (n)) / \log (n)=1=$ $\beta+2$ for $\beta=-1$ and thus (16) holds.

To see that (16) holds for $\beta>-1$ as well, multiply $n$ in the above estimate and observe that there is some constant $\tilde{C}>0$ such that, for all $n$ sufficiently large,

$$
\sum_{k=\tau_{0}}^{n-1}(n-k) k^{\beta} \geq \tilde{C} n^{\beta+2}
$$

This clearly implies that $\liminf \operatorname{in}_{n \rightarrow \infty} \log \left(\sum_{k=\tau_{0}}^{n-1}(n-k) k^{\beta}\right) / \log (n) \geq \beta+2$. For the opposite inequality, observe that, for $\beta>-1$,

$$
\frac{1}{n} \sum_{k=\tau_{0}}^{n-1}(n-k) k^{\beta} \leq \sum_{k=\tau_{0}}^{n-1} k^{\beta} \leq \int_{\tau_{0}-1}^{n} k^{\beta} \mathrm{d} k \leq(\beta+1)^{-1} n^{\beta+1}
$$

which implies that $\lim \sup _{n \rightarrow \infty} \log \left(\sum_{k=\tau_{0}}^{n-1}(n-k) k^{\beta}\right) / \log (n) \leq \beta+2$.

A first consequence of Lemma 1 is the following observation regarding the asymptotic behavior of the autocorrelation.

Lemma 2 Given $\left(X_{t}\right)_{t \in[0, \infty[}$, for any $s \geq \frac{1}{2}$, it holds that

$$
\limsup _{\tau \rightarrow \infty} \frac{c(\tau)}{\tau^{2 s-2}} \geq 0
$$


Proof Assume that the left-hand side of the inequality above is strictly less than 0 for some $s \geq \frac{1}{2}$. Then there exists some constant $C>0$ and some $\tau_{0} \in \mathbb{N}$ such that

$$
c(\tau) \leq-C \tau^{2 s-2}
$$

holds for all $\tau \geq \tau_{0}$. From (14) it is inferred that, for any $n \geq \tau_{0}$,

$$
\begin{aligned}
0 & \leq \frac{\operatorname{Var}\left(X_{n}\right)}{\operatorname{Var}\left(X_{1}\right)}=n+2 \sum_{k=1}^{n-1}(n-k) c(k) \\
& =n+2 \cdot\left(\sum_{k=1}^{\tau_{0}-1}(n-k) c(k)+\sum_{k=\tau_{0}}^{n-1}(n-k) c(k)\right) \\
& \leq\left(1+2 \sum_{k=1}^{\tau_{0}-1}|c(k)|\right) \cdot n-2 C \sum_{k=\tau_{0}}^{n-1}(n-k) k^{2 s-2} .
\end{aligned}
$$

Noting that the term in parentheses is a constant, Lemma 1 implies that the second summand in this expression dominates the first one, provided $n$ is large enough. Hence for large $n$ the last expression is negative, a contradiction.

Under the stated assumptions, Lemma 2 shows in particular that, if the limit in (A5) is assumed to exist (for some $H \geq 0.5$ ), then it cannot be negative. The next statement clarifies that assuming the existence of the limit (A5) for some $H \in \mathbb{R}$ implies that $H$ is the Hurst exponent of the process.

Lemma 3 Assume for $\left(X_{t}\right)_{t \in[0, \infty[}$ that the limit in (A5) exists and equals $C$ for some constants $H \in \mathbb{R}$ and $C \neq 0$. Then for any $s>H, \lim _{\tau \rightarrow \infty} \frac{c(\tau)}{\tau^{2 s-2}}=0$, and, $\lim _{\tau \rightarrow \infty} \frac{c(\tau)}{\tau^{2 s-2}} \in\{+\infty,-\infty\}$, for any $s<H$, where the sign corresponds to the sign of $C$. In particular, the Hurst exponent of $\left(X_{t}\right)_{t \in[0, \infty[}$ (as defined in (5)) exists and equals $H$. Consequently, the constant $C$ is positive.

Proof Observe that, for any $s \in \mathbb{R}$,

$$
\frac{c(\tau)}{\tau^{2 s-2}}=\frac{c(\tau)}{\tau^{2 H-2}} \cdot \tau^{2(H-s)} .
$$

Now the assumptions imply that the first factor on the right converges (to the constant $C \neq 0$ ), as $\tau \rightarrow \infty$, while the second factor tends to zero for $s>H$ and to $+\infty$ for $s<H$. From this the first assertion is obvious. It is an immediate consequence that the number $H$ is the common value of the lower and upper Hurst exponents of $\left(X_{t}\right)_{t \in[0, \infty[}$ as defined in (13), i.e., $H=\underline{H}=\bar{H}$. Moreover, the Hurst exponent in the sense of (5) also exists and equals $H$. (The existence of the limit in (A5) implies that for any $\delta$ such that $0<\delta<C$, there is some $\tau_{0}$ such that $(|C|-\delta) \cdot \tau^{2 H-2} \leq|c(\tau)| \leq(|C|+\delta) \cdot \tau^{2 H-2}$ for all $\tau \geq \tau_{0}$. Taking logarithms in this inequality and dividing by $\log (\tau)$ yields

$$
2 H-2+\frac{\log (|C|-\delta)}{\log (\tau)} \leq \frac{\log (|c(\tau)|)}{\log (\tau)} \leq 2 H-2+\frac{\log (|C|+\delta) \mid)}{\log (\tau)}
$$


Letting $\tau \rightarrow \infty$, the expressions on the left and on the right converge to $2 H-2$, from which the existence of the limit in (5) is clear.)

The next statement gives a general upper bound for the asymptotics of the variance $\operatorname{Var}\left(X_{\tau}\right)$ as $\tau \rightarrow \infty$ of a process $\left(X_{t}\right)_{t \in[0, \infty[}$ in terms of its upper Hurst exponent.

Proposition 2 If $H$ is the upper Hurst exponent of $\left(X_{t}\right)_{t \in[0, \infty[}$ (as defined in (13)), then

$$
\limsup _{n \rightarrow \infty} \frac{\log \left(\operatorname{Var}\left(X_{n}\right)\right)}{\log (n)} \leq \max \{1,2 H\} .
$$

Proof Observe that due to the relation (14), it is enough to show that

$$
\limsup _{n \rightarrow \infty} \frac{\log \left(n+2 \sum_{k=1}^{n-1}(n-k) c(k)\right)}{\log (n)} \leq \max \{1,2 H\} .
$$

Let $\varepsilon>0$. The definition of the upper Hurst exponent (13) implies that there exists some $\tau_{0} \in \mathbb{N}$ such that

$$
|c(\tau)| \leq \tau^{2 H-2+\epsilon}
$$

holds for all $\tau \geq \tau_{0}$. It is inferred that, for $n>\tau_{0}$,

$$
\begin{aligned}
n+2 \sum_{k=1}^{n-1}(n-k) c(k) & \leq n+2 \sum_{k=1}^{\tau_{0}-1}(n-k)|c(k)|+2 \sum_{k=\tau_{0}}^{n-1}(n-k)|c(k)| \\
& \leq n\left(1+2 \sum_{k=1}^{\tau_{0}-1}|c(k)|\right)+2 \sum_{k=\tau_{0}}^{n-1}(n-k) k^{2 H-2+\varepsilon}
\end{aligned}
$$

where the expression in parentheses is a constant independent of $n$. Let us denote it by $E$. Applying the formula $\log (a+b) \leq b / a+\log (a)$ (which is valid for $a, a+b>0$ ), with $a:=2 \sum_{k=\tau_{0}}^{n-1}(n-k) k^{2 H-2+\varepsilon}$ and $b:=n \cdot E$ it is inferred that

$$
\begin{aligned}
& \limsup _{n \rightarrow \infty} \frac{\log \left(n+2 \sum_{k=1}^{n-1}(n-k) c(k)\right)}{\log (n)} \\
& \leq \limsup _{n \rightarrow \infty} \frac{\log \left(n \cdot E+2 \sum_{k=\tau_{0}}^{n-1}(n-k) k^{2 H-2+\varepsilon}\right)}{\log (n)} \\
& \leq \limsup _{n \rightarrow \infty}\left(\frac{n \cdot E}{\log (n) \cdot 2 \sum_{k=\tau_{0}}^{n-1}(n-k) k^{2 H-2+\varepsilon}}\right. \\
& \left.\quad+\frac{\log \left(2 \sum_{k=\tau_{0}}^{n-1}(n-k) k^{2 H-2+\varepsilon}\right)}{\log (n)}\right) .
\end{aligned}
$$


Now observe that for $H \geq 0.5$, by the inequality in Lemma 1 , the first quotient vanishes as $n \rightarrow \infty$, and by (16) in Lemma 1 , the second quotient converges to $2 H+\varepsilon$. Since this estimate holds for any $\varepsilon>0$, the assertion follows for any $H \geq 0.5$ by letting $\varepsilon \searrow 0$.

For $H<0.5$, the summand $a$ from above can be bounded as follows

$$
a \leq 2 n^{1+\varepsilon} \sum_{k=\tau_{0}}^{n-1} k^{2 H-2} \leq 2 n^{1+\varepsilon} \int_{\tau_{0}-1}^{n} k^{2 H-2} \mathrm{~d} k \leq n^{1+\varepsilon} \frac{2\left(\tau_{0}-1\right)^{2 H-1}}{1-2 H}
$$

Since this holds for any $\varepsilon>0$ (and since the summand $b$ grows like $n$, as $n \rightarrow \infty$ ), one concludes that 1 is an upper bound for the limes superior in (17), as asserted for $H<0.5$. This completes the proof.

Proposition 3 Let $H \geq 0.5$ and $C>0$. Assume that for $\left(X_{t}\right)_{t \in[0, \infty[}$ the limit in (A5) exists and equals $C$. Then $H$ is the Hurst exponent of $\left(X_{t}\right)_{t \in[0, \infty[}$ (as defined in (5)) and

$$
\lim _{n \rightarrow \infty} \frac{\log \left(\operatorname{Var}\left(X_{n}\right)\right)}{\log (n)}=2 H
$$

Proof By Lemma 3, $H$ is indeed the Hurst exponent of $\left(X_{t}\right)_{t \in[0, \infty[}$. In view of Proposition 2 and the relation (14), the only thing that remains to be shown is that

$$
L:=\liminf _{n \rightarrow \infty} \frac{\log \left(n+2 \sum_{k=1}^{n-1}(n-k) c(k)\right)}{\log (n)} \geq 2 H .
$$

Since the limit in (A5) is assumed to equal $C>0$, there exists $\tau_{0} \in \mathbb{N}$ such that

$$
\frac{C}{2} \tau^{2 H-2} \leq c(\tau)
$$

holds for all $\tau \geq \tau_{0}$. In particular, $c(\tau)$ will be positive for $\tau>\tau_{0}$.

This relation implies that, for any $n \in \mathbb{N}$,

$$
\begin{aligned}
n+2 \sum_{k=1}^{n-1}(n-k) c(k) & =n+2 \sum_{k=1}^{\tau_{0}-1}(n-k) c(k)+2 \sum_{k=\tau_{0}}^{n-1}(n-k) c(k) \\
& \geq-\left|n+2 \sum_{k=1}^{\tau_{0}-1}(n-k) c(k)\right|+C \sum_{k=\tau_{0}}^{n-1}(n-k) k^{2 H-2} \\
& \geq-n \cdot\left(1+2 \sum_{k=1}^{\tau_{0}-1}|c(k)|\right)+C \sum_{k=\tau_{0}}^{n-1}(n-k) k^{2 H-2}
\end{aligned}
$$

Now Lemma 1 ensures that there exists a $\tau_{1}$ such that the last expression is positive for all $n \geq \tau_{1}$. Employing the formula $\log (a+b) \geq b / a-(b / a)^{2} / 2+\log (a)$, which is valid for $a, a+b>0$, allows one to conclude that 


$$
\begin{aligned}
L \geq & \liminf _{n \rightarrow \infty} \frac{\log \left(C \sum_{k=\tau_{0}}^{n-1}(n-k) k^{2 H-2}-E n\right)}{\log (n)} \\
& \geq \liminf _{n \rightarrow \infty} \frac{1}{\log (n)}\left(\frac{-E \cdot n}{C \sum_{k=\tau_{0}}^{n-1}(n-k) k^{2 H-2}}-\frac{E^{2} \cdot n^{2}}{2\left(C \sum_{k=\tau_{0}}^{n-1}(n-k) k^{2 H-2}\right)^{2}}\right. \\
& \left.+\log \left(C \sum_{k=\tau_{0}}^{n-1}(n-k) \cdot k^{2 H-2}\right)\right) .
\end{aligned}
$$

By the inequality in Lemma 1, the first two summands are bounded and therefore, when divided by $\log (n)$, they vanish as $n \rightarrow \infty$. Furthermore, by (16), the third summand divided by $\log (n)$ converges to $2 H$. Note that for the application of Lemma 1 the assumption $H \geq 0.5$ was used.

After these preparations a proof of Theorem 1 can be provided.

Proof of Theorem 1 Under the assumptions of the theorem, Proposition 3 applies. Thus, $\lim _{n \rightarrow \infty} \frac{\log _{2}\left(\operatorname{Var}\left(X_{n}\right)\right)}{\log _{2}(n)}$ exists and equals $2 H$. One can compute this limit by passing to any subsequence $\left(\tau_{n}\right)_{n \in \mathbb{N}} \subseteq \mathbb{N}$ diverging to $\infty$. Choose $\tau_{n}=2^{n}$. Then

$$
\begin{aligned}
H & =\frac{1}{2} \lim _{n \rightarrow \infty} \frac{\log _{2}\left(\operatorname{Var}\left(X_{2^{n}}\right)\right)}{\log _{2}\left(2^{n}\right)} \\
& =\frac{1}{2} \lim _{n \rightarrow \infty} \frac{1}{n} \log _{2}\left(\operatorname{Var}\left(X_{2^{n}}\right)\right) \\
& =\frac{1}{2} \lim _{n \rightarrow \infty} \frac{1}{n}\left[\log _{2}\left(\operatorname{Var}\left(X_{2^{n}}\right)\right)-\log _{2}\left(\operatorname{Var}\left(X_{1}\right)\right)\right] \\
& =\frac{1}{2} \lim _{n \rightarrow \infty} \frac{1}{n} \sum_{i=0}^{n-1} \log _{2}\left(\frac{\operatorname{Var}\left(X_{2^{i+1}}\right)}{\operatorname{Var}\left(X_{2^{i}}\right)}\right) .
\end{aligned}
$$

Together with Proposition 1 and the Stolz-Cesáro theorem, this implies that

$$
\begin{aligned}
\liminf _{\tau \rightarrow \infty} h(p(\tau)) & \leq \liminf _{n \rightarrow \infty} h\left(p\left(2^{n}\right)\right)=\frac{1}{2} \liminf _{n \rightarrow \infty} \log _{2}\left(\frac{\operatorname{Var}\left(X_{2^{n+1}}\right)}{\operatorname{Var}\left(X_{2^{n}}\right)}\right) \\
& \leq \frac{1}{2} \liminf _{n \rightarrow \infty} \frac{1}{n} \sum_{i=0}^{n-1} \log _{2}\left(\frac{\operatorname{Var}\left(X_{2^{i+1}}\right)}{\operatorname{Var}\left(X_{2^{i}}\right)}\right)=H \\
& =\frac{1}{2} \limsup _{n \rightarrow \infty} \frac{1}{n} \sum_{i=0}^{n-1} \log _{2}\left(\frac{\operatorname{Var}\left(X_{2^{i+1}}\right)}{\operatorname{Var}\left(X_{2^{i}}\right)}\right) \\
& \leq \frac{1}{2} \limsup _{n \rightarrow \infty} \log _{2}\left(\frac{\operatorname{Var}\left(X_{2^{n+1}}\right)}{\operatorname{Var}\left(X_{2^{n}}\right)}\right) \\
& =\limsup _{n \rightarrow \infty} h\left(p\left(2^{n}\right)\right) \leq \limsup _{\tau \rightarrow \infty} h(p(\tau)) .
\end{aligned}
$$


So, in particular, if the $\operatorname{limit}_{\tau \rightarrow \infty} h(p(\tau))$ exists, it must be equal to $H$, which completes the proof of the theorem.

Recall that it was assumed in Theorem 1 that the $\operatorname{limit}_{\tau \rightarrow \infty} h(p(\tau))$ exists in order to conclude that it must be equal to $H$. The last sequence of inequalities in the above proof shows that, alternatively, one can impose the weaker assumption that $\lim _{n \rightarrow \infty} h\left(p\left(2^{n}\right)\right)$ exists in order to prove the (slightly weaker) statement that $\lim _{n \rightarrow \infty} h\left(p\left(2^{n}\right)\right)=H$ holds.

\section{References}

Abry P, Flandrin P, Taqqu MS, Veitch D (2000) Self-similarity and long-range dependence through the wavelet lens. In: Doukhan P, Oppenheim G, Taqqu MS (eds) Theory and applications of long-range dependence. Birkhäuser, Boston, pp 527-556

Amigó JM (2010) Permutation complexity in dynamical systems. Ordinal patterns, permutation entropy and all that. Springer, Dordrecht. ISBN 978-3-642-04083-2/hbk; 978-3-642-04084-9/ebook

Amigó JM, Keller K, Unakafova VA (2014) Ordinal symbolic analysis and its application to biomedical recordings. Philos Trans R Soc Lond Ser A 373(2034):20140091

Arcones MA (1994) Limit theorems for nonlinear functionals of a stationary Gaussian sequence of vectors. Ann Probab 22(4):2242-2274

Bandis S, Lumsden A, Barton N (1983) Fundamentals of rock joint deformation. Int J Rock Mech Min Sci Geomech 20(6):249-268

Bandt C, Shiha F (2007) Order patterns in time series. J Time Ser Anal 28(5):646-665

Barton N (1973) Review of a new shear-strength criterion for rock joints. Eng Geol 7(4):287-332

Barton N, Choubey V (1977) The shear strength of rock joints in theory and practice. Rock Mech 10(1):1-54

Bistacci A, Griffith WA, Smith SAF, Di Toro G, Jones R, Nielsen S (2011) Fault roughness at seismogenic depths from LIDAR and photogrammetric analysis. Pure Appl Geophys 168:2345-2363

Boutt DF, Grasselli G, Fredrich JT, Cook BK, Williams JR (2006) Trapping zones: the effect of fracture roughness on the directional anisotropy of fluid flow and colloid transport in a single fracture. Geophys Res Lett 33(21):L21402

Brodsky EE, Kirkpatrick JD, Candela T (2016) Constraints from fault roughness on the scale-dependent strength of rocks. Geology 44(1):19-22

Brown SR (1987) Fluid flow through rock joints: the effect of surface roughness. J Geophys Res Solid Earth 92:1337-1347

Candela T, Renard F, Klinger Y, Mair K, Schmittbuhl J, Brodsky EE (2012) Roughness of fault surfaces over nine decades of length scales. J Geophys Res Solid Earth 117(B8):1-30

Chang S, Pihl G, Essigmann M (1951) Representations of speech sounds and some of their statistical properties. Proc IRE 39:147-153

Coeurjolly JF (2000) Simulation and identification of the fractional Brownian motion: a bibliographical and comparative study. J Stat Softw 5(7):1-53

Corradetti A, McCaffrey K, De Paola N, Tavani S (2017) Evaluating roughness scaling properties of natural active fault surfaces by means of multi-view photogrammetry. Tectonophysics 717:599-606

Corradetti A, Zambrano M, Tavani S, Tondi E, Seers TD (2020) The impact of weathering upon the roughness characteristics of a splay of the active fault system responsible for the massive 2016 seismic sequence of the central Apennines, Italy. Geol Soc Am Bull 130:885-896

Dong J, Ju Y (2020) Quantitative characterization of single-phase flow through rough-walled fractures with variable apertures. Geomech Geophys Geo-Energy Geo-Resour 6:42

Doob J (1935) The limiting distributions of certain statistics. Ann Math Stat 6(3):160-169

Dou Z, Sleep B, Zhan H, Zhou Z, Wang J (2019) Multiscale roughness influence on conservative solute transport in self-affine fractures. Int J Heat Mass Transf 133:606-618

El-Soudani SM (1978) Profilometric analysis of fractures. Metallography 11(3):247-336

Ewing G, Taylor J (1969) Computer recognition of speech using zero-crossing information. IEEE Trans Audio Electroacoust 17:37-40 
Fischer C, Gaupp R, Dimke M, Sill O (2007) A 3d high resolution model of bounding surfaces in aeolianfluvial deposits: an outcrop analogue study from the Permian Rotliegend, northern Germany. J Pet Geol 30(3):257-273

Flandrin P (1992) Wavelet analysis and synthesis of fractional Brownian motion. IEEE Trans Inf Theory 38:910-917

Gneiting T, Schlather M (2004) Stochastic models that separate fractal dimension and the Hurst effect. SIAM Rev 46(2):269-282

Gutjahr T (2021) Hurst exponent based on change probabilities. MATLAB central file exchange. https://www.mathworks.com/matlabcentral/fileexchange/80008-hurst-exponent-based-on-changeprobabilities. Accessed 25 Feb 2021

Hale S, Naab C, Butscher C, Blum P (2020) Method comparison to determine hydraulic apertures of natural fractures. Rock Mech Rock Eng 53(3):1467-1476

Heidsiek M, Butscher C, Blum P, Fischer C (2020) Small-scale diagenetic facies heterogeneity controls porosity and permeability pattern in reservoir sandstones. Environ Earth Sci 79(18):425

Ho HC, Sun TC (1987) A central limit theorem for non-instantaneous filters of a stationary gaussian process. J Multivar Anal 22(1):144-155

Hsiung SM, Ghosh A, Chowdhury AH (1995) On natural rock joint profile characterization using selfaffine fractal approach. In: Daemen J, Schultz R (eds) Proceedings of the 35th U.S. symposium on rock mechanics. Balkema, Rotterdam, pp 681-687

Huang SL, Oelfke SM, Speck RC (1992) Applicability of fractal characterization and modelling to rock joint profiles. Int Rock Mech Min Sci Geomech Abstr 29(2):89-98

Issa MA, Issa MA, Islam MS, Chudnovsky A (2003) Fractal dimension-a measure of fracture roughness and toughness of concrete. Eng Fract Mech 70(1):125-137

ISTerre (2021) Institut des sciences de la terre. Fault slip surface data. https://www.isterre.fr/french/ recherche-observation/equipes/mecanique-des-failles/moyens-et-outils/article/donnees.html. Accessed 25 Feb 2021

Jiang Y, Li B, Tanabashi Y (2006) Estimating the relation between surface roughness and mechanical properties of rock joints. Int J Rock Mech Min Sci 43(6):837-846

Kottwitz MO, Popov AA, Baumann TS, Kaus BJP (2020) The hydraulic efficiency of single fractures: correcting the cubic law parameterization for self-affine surface roughness and fracture closure. Solid Earth 11(3):947-957

Lee JJ, Bruhn RL (1996) Structural anisotropy of normal fault surfaces. J Struct Geol 18(8):1043-1059

Li X, Jiang Z, Couples GG (2019) A stochastic method for modelling the geometry of a single fracture: spatially controlled distributions of aperture, roughness and anisotropy. Transp Porous Media 128(2):797-819

Liu R, He M, Huang N, Jiang Y, Yu L (2020) Three-dimensional double-rough-walled modeling of fluid flow through self-affine shear fractures. J Rock Mech Geotech Eng 12(1):41-49

Magsipoc E, Zhao Q, Grasselli G (2020) 2d and 3d roughness characterization. Rock Mech Rock Eng 53(3):1495-1519

Malinverno A (1990) A simple method to estimate the fractal dimension of self-affine series. Geophys Res Lett 17(11):1953-1956

Mandelbrot BB (1985) Self-affine fractals and fractal dimension. Physica Scr 32(4):257-260

McClean CJ, Evans IS (2002) Apparent fractal dimensions from continental scale digital elevation models using variogram methods. Trans GIS 4(4):361-378

Morgan SP, Johnson CA, Einstein HH (2013) Cracking processes in barre granite: fracture process zones and crack coalescence. Int J Fract 180(2):177-204

Myers NO (1962) Characterization of surface roughness. Wear 5(3):182-189

Odling NE (1994) Natural fracture profiles, fractal dimension and joint roughness coefficients. Rock Mech Rock Eng 27(3):135-153

Poon CY, Sayles RS, Jones TA (1992) Surface measurement and fractal characterization of naturally fractured rocks. J Phys D Appl Phys 25(8):1269-1275

Power WL, Durham WB (1997) Topography of natural and artificial fractures in granitic rocks: implications for studies of rock friction and fluid migration. Int J Rock Mech Min Sci 34(6):979-989

Power WL, Tullis TE (1991) Euclidean and fractal models for the description of rock surface roughness. J Geophys Res Solid Earth 96:415-424

Renard F, Voisin C, Marsan D, Schmittbuhl J (2006) High resolution 3d laser scanner measurements of a strike-slip fault quantify its morphological anisotropy at all scales. Geophys Res Lett 33(4):L04305 
Renard F, Candela T, Bouchaud E (2013) Constant dimensionality of fault roughness from the scale of micro-fractures to the scale of continents. Geophys Res Lett 40(1):83-87

Roux S, Schmittbuhl J, Vilotte JP, Hansen A (1993) Some physical properties of self-affine rough surfaces. Europhys Lett 23(4):277-282

Sagy A, Brodsky EE, Axen GJ (2007) Evolution of fault-surface roughness with slip. Geology 35(3):283286

Schmittbuhl J, Gentier S, Roux S (1993) Field measurements of the roughness of fault surfaces. Geophys Res Lett 20(8):639-641

Seybold HJ, Carmona HA, Filho FAL, Araújo AD, Filho FN, Andrade JS (2020) Flow through threedimensional self-affine fractures. Phys Rev Fluids 5(10):104101

Siman-Tov S, Aharonov E, Sagy A, Emmanuel S (2013) Nanograins form carbonate fault mirrors. Geology 41(6):703-706

Sinn M, Keller K (2011a) Covariances of zero crossings in gaussian processes. Theory Prob Appl 55(3):485504

Sinn M, Keller K (2011b) Estimation of ordinal pattern probabilities in Gaussian processes with stationary increments. Comput Stat Data Anal 55(4):1781-1790

Stigsson M, Mas Ivars D (2019) A novel conceptual approach to objectively determine JRC using fractal dimension and asperity distribution of mapped fracture traces. Rock Mech Rock Eng 52(4):1041-1054

Thompson ME, Brown SR (1991) The effect of anisotropic surface roughness on flow and transport in fractures. J Geophys Res Solid Earth 96:21923-21932

Tsang YW, Witherspoon PA (1983) The dependence of fracture mechanical and fluid flow properties on fracture roughness and sample size. J Geophys Res 88:2359

Vogler D, Walsh SDC, Bayer P, Amann F (2017) Comparison of surface properties in natural and artificially generated fractures in a crystalline rock. Rock Mech Rock Eng 50(11):2891-2909

Xie H, Wang JA, Kwaśniewski M (1999) Multifractal characterization of rock fracture surfaces. Int J Rock Mech Min Sci 36(1):19-27

Yu X, Regenauer-Lieb K, Tian FB (2020) Effects of surface roughness and derivation of scaling laws on gas transport in coal using a fractal-based lattice Boltzmann method. Fuel 259:116229

Zambrano M, Pitts AD, Salama A, Volatili T, Giorgioni M, Tondi E (2019) Analysis of fracture roughness control on permeability using SfM and fluid flow simulations: implications for carbonate reservoir characterization. Geofluids 2019:4132386

Zanin M, Zunino L, Rosso OA, Papo D (2012) Permutation entropy and its main biomedical and econophysics applications: a review. Entropy 14(8):1553-1577

Zhao Z, Li B, Jiang Y (2014) Effects of fracture surface roughness on macroscopic fluid flow and solute transport in fracture networks. Rock Mech Rock Eng 47(6):2279-2286

Zimmerman R, Bodvarsson G (1996) Hydraulic conductivity of rock fractures. Transp Porous Media 23(1):1-30 